\title{
Higher-order effects on the incompressibility of isospin asymmetric nuclear matter
}

\author{
Lie-Wen Chen, ${ }^{1,2}$ Bao-Jun Cai, ${ }^{1}$ Che Ming Ko,${ }^{3}$ Bao-An Li,${ }^{4}$ Chun Shen, ${ }^{1}$ and Jun $\mathrm{Xu}^{3}$ \\ ${ }^{1}$ Department of Physics, Shanghai Jiao Tong University, Shanghai 200240, People's Republic of China \\ ${ }^{2}$ Center of Theoretical Nuclear Physics, National Laboratory of Heavy Ion Accelerator, Lanzhou 730000, People's Republic of China \\ ${ }^{3}$ Cyclotron Institute and Physics Department, Texas A\&M University, College Station, Texas 77843-3366, USA \\ ${ }^{4}$ Department of Physics, Texas A\&M University-Commerce, Commerce, Texas 75429-3011, USA
}

(Received 27 May 2009; published 30 July 2009)

\begin{abstract}
Analytical expressions for the saturation density of asymmetric nuclear matter as well as its binding energy and incompressibility at saturation density are given up to fourth order in the isospin asymmetry $\delta=\left(\rho_{n}-\rho_{p}\right) / \rho$ using 11 characteristic parameters defined by the density derivatives of the binding energy per nucleon of symmetric nuclear matter, the symmetry energy $E_{\text {sym }}(\rho)$, and the fourth-order symmetry energy $E_{\text {sym }, 4}(\rho)$ at normal nuclear density $\rho_{0}$. Using an isospin- and momentum-dependent modified Gogny interaction (MDI) and the Skyrme-Hartree-Fock (SHF) approach with 63 popular Skyrme interactions, we have systematically studied the isospin dependence of the saturation properties of asymmetric nuclear matter, particularly the incompressibility $K_{\text {sat }}(\delta)=K_{0}+K_{\text {sat }, 2} \delta^{2}+K_{\text {sat }, 4} \delta^{4}+O\left(\delta^{6}\right)$ at saturation density. Our results show that the magnitude of the higher order $K_{\mathrm{sat}, 4}$ parameter is generally small compared to that of the $K_{\mathrm{sat}, 2}$ parameter. The latter essentially characterizes the isospin dependence of the incompressibility at saturation density and can be expressed as $K_{\mathrm{sat}, 2}=K_{\text {sym }}-6 L-\frac{J_{0}}{K_{0}} L$, where $L$ and $K_{\text {sym }}$ represent, respectively, the slope and curvature parameters of the symmetry energy at $\rho_{0}$ and $J_{0}$ is the third-order derivative parameter of symmetric nuclear matter at $\rho_{0}$. Furthermore, we have constructed a phenomenological modified Skyrme-like (MSL) model that can reasonably describe the general properties of symmetric nuclear matter and the symmetry energy predicted by both the MDI model and the SHF approach. The results indicate that the higher order $J_{0}$ contribution to $K_{\text {sat, } 2}$ generally cannot be neglected. In addition, it is found that there exists a nicely linear correlation between $K_{\text {sym }}$ and $L$ as well as between $J_{0} / K_{0}$ and $K_{0}$. These correlations together with the empirical constraints on $K_{0}, L, E_{\text {sym }}\left(\rho_{0}\right)$, and the nucleon effective mass lead to an estimate of $K_{\text {sat }, 2}=-370 \pm 120 \mathrm{MeV}$.
\end{abstract}

DOI: 10.1103/PhysRevC.80.014322

PACS number(s): 21.65.Mn, 21.30.Fe, 21.65.Cd, 21.65.Ef

\section{INTRODUCTION}

The equation of state (EOS) of nuclear matter is one of central issues in nuclear physics. For cold nuclear matter, the EOS is usually defined as the binding energy per nucleon as a function of the density, from which information on other thermodynamic properties of nuclear matter, such as its pressure and incompressibility, can be obtained. With the establishment or construction of many radioactive beam facilities around the world, such as the Cooling Storage Ring (CSR) facility at HIRFL in China [1], the Radioactive Ion Beam (RIB) Factory at RIKEN in Japan [2], the FAIR/GSI in Germany [3], SPIRAL2/GANIL in France [4], and the Facility for Rare Isotope Beams (FRIB) in the United States [5], it is possible in terrestrial laboratories to explore the EOS of isospin asymmetric nuclear matter under the extreme condition of large isospin asymmetry, especially with regard to the density dependence of the nuclear symmetry energy. This knowledge, especially the latter, is important for understanding not only the structure of radioactive nuclei, the reaction dynamics induced by rare isotopes, and the liquid-gas phase transition in asymmetric nuclear matter but also many critical issues in astrophysics [6-15].

For symmetric nuclear matter with equal fractions of neutrons and protons, its EOS is relatively well determined after about more than 30 years of studies by the nuclear physics community. In particular, the incompressibility of symmetric nuclear matter at its saturation density $\rho_{0}$ has been determined to be $240 \pm 20 \mathrm{MeV}$ from analyses of the nuclear giant monopole resonances (GMR) [16-24], and its EOS at densities of $2 \rho_{0}<\rho<5 \rho_{0}$ has also been constrained by measurements of collective flows in nucleus-nucleus collisions [8] and of subthreshold kaon production $[25,26]$ in relativistic nucleus-nucleus collisions. In contrast, the EOS of asymmetric nuclear matter, especially the density dependence of the nuclear symmetry energy, is largely unknown. Although the nuclear symmetry energy at $\rho_{0}$ is known to be around $30 \mathrm{MeV}$ from the empirical liquid-drop mass formula [27,28], its values at other densities, especially at supra-saturation densities, are poorly known [6,7]. Various microscopic and phenomenological models, such as the relativistic Dirac-Brueckner-HartreeFock (DBHF) [29-35] and the nonrelativistic BruecknerHartree-Fock (BHF) [36-39] approach, the relativistic mean-field (RMF) model based on nucleon-meson interactions $[12,40-42]$, and the nonrelativistic mean-field model based on Skyrme-like interactions [43-51], have been used to study the isospin-dependent properties of asymmetric nuclear matter, such as the nuclear symmetry energy, the nuclear symmetry potential, and the isospin-splitting of the nucleon effective masses, but the predicted results vary widely. In fact, even the sign of the symmetry energy above $3 \rho_{0}$ is still uncertain $[52,53]$. The theoretical uncertainties are mainly due to the lack of knowledge about the isospin dependence of in-medium nuclear effective interactions and the limitations in the techniques for solving the nuclear many-body problem. 
Heavy-ion collisions, especially those induced by neutronrich nuclei, provide a unique tool to investigate the EOS of asymmetric nuclear matter, especially the density dependence of the nuclear symmetry energy. During the past decade, significant progress has indeed been made both experimentally and theoretically on constraining the behavior of the symmetry energy at subsaturation density using heavy-ion reactions [50,54-60] (see Ref. [15] for the most recent review). More recently, the IBUU04 transport model analysis of the FOPI data on the $\pi^{-} / \pi^{+}$ratio in central heavy-ion collisions at SIS/GSI [61] energies suggests a very soft symmetry energy at supra-saturation densities [62]. Information on the density dependence of the nuclear symmetry energy has also been obtained from the structure of finite nuclei and their excitations, such as the mass data [63], neutron skin in heavy nuclei [64], giant dipole resonances [65], pygmy dipole resonance [66], and so on. These studies have significantly improved our understanding of the EOS of asymmetric nuclear matter.

The incompressibility of asymmetric nuclear matter at its saturation density is a basic quantity to characterize its EOS. Since this quantity is largely undetermined, any constraint imposed on it would be extremely important. In the present work, we study the isospin dependence of the properties of asymmetric nuclear matter, including the saturation density as well as the binding energy and incompressibility at saturation density. In particular, we derive analytical expressions for these quantities up to the fourth order in the isospin asymmetry $\delta=\left(\rho_{n}-\rho_{p}\right) / \rho$ and investigate the higher order isospin asymmetry effects on the properties of asymmetric nuclear matter. The incompressibility of asymmetric nuclear matter at its saturation density can be written as $K_{\text {sat }}(\delta)=K_{0}+$ $K_{\text {sat }, 2} \delta^{2}+K_{\text {sat }, 4} \delta^{4}+O\left(\delta^{6}\right)$ with $K_{\text {sat }, 2}=K_{\text {sym }}-6 L-\frac{J_{0}}{K_{0}} L$, where $L$ and $K_{\text {sym }}$ represent, respectively, the slope and curvature parameters of the symmetry energy at $\rho_{0}$ and $J_{0}$ is the third-order derivative parameter of symmetric nuclear matter at $\rho_{0}$. Therefore, the higher order effects on $K_{\text {sat }}(\delta)$ also include the third-order density derivative parameter $J_{0}$. Our results indicate that higher order isospin asymmetry effects on the incompressibility are usually not important but the higher order $J_{0}$ contribution generally cannot be neglected. Furthermore, we construct a phenomenological modified Skyrme-like model that can reasonably describe the general properties of symmetric nuclear matter and the symmetry energy predicted by both the momentum-dependent modified Gogny interaction model and the Skyrme-Hartree-Fock (SHF) approach. We find that there exists a nicely linear correlation between $K_{\text {sym }}$ and $L$ as well as between $J_{0} / K_{0}$ and $K_{0}$. These correlations together with the empirical constraints on $K_{0}, L$, and $E_{\text {sym }}\left(\rho_{0}\right)$, and the nucleon effective mass lead to an estimate of $K_{\text {sat, } 2}=-370 \pm$ $120 \mathrm{MeV}$.

The paper is organized as follows. In Sec. II, we discuss the general properties of asymmetric nuclear matter, and then give analytical expressions up to fourth-order terms in isospin asymmetry $\delta$ for the saturation density of asymmetric nuclear matter as well as its binding energy and incompressibility at saturation density. We then briefly introduce in Sec. III the three models used in the present paper (i.e., the isospin- and momentum-dependent modified Gogny interaction model, the
SHF approach, and the phenomenological modified Skyrmelike model). The results and discussions are presented in Sec. IV. A summary is then given in Sec. V. For completeness, derivations of some important formula shown in Sec. II are briefly described in the Appendix.

\section{SATURATION PROPERTIES OF ASYMMETRIC NUCLEAR MATTER}

\section{A. Equation of state of asymmetric nuclear matter}

The EOS of isospin asymmetric nuclear matter, given by its binding energy per nucleon, can be generally expressed as a power series in the isospin asymmetry $\delta=\left(\rho_{n}-\rho_{p}\right) / \rho$, where $\rho=\rho_{n}+\rho_{p}$ is the baryon density with $\rho_{n}$ and $\rho_{p}$ denoting the neutron and proton densities, respectively. To the fourth order in isospin asymmetry, it is written as

$$
E(\rho, \delta)=E_{0}(\rho)+E_{\mathrm{sym}}(\rho) \delta^{2}+E_{\mathrm{sym}, 4}(\rho) \delta^{4}+O\left(\delta^{6}\right),
$$

where $E_{0}(\rho)=E(\rho, \delta=0)$ is the binding energy per nucleon of symmetric nuclear matter and

$$
\begin{gathered}
E_{\mathrm{sym}}(\rho)=\left.\frac{1}{2 !} \frac{\partial^{2} E(\rho, \delta)}{\partial \delta^{2}}\right|_{\delta=0}, \\
E_{\mathrm{sym}, 4}(\rho)=\left.\frac{1}{4 !} \frac{\partial^{4} E(\rho, \delta)}{\partial \delta^{4}}\right|_{\delta=0} .
\end{gathered}
$$

The term $E_{\text {sym }}(\rho)$ is the so-called nuclear symmetry energy and $E_{\mathrm{sym}, 4}(\rho)$ is the fourth-order coefficient, which is called here the fourth-order nuclear symmetry energy. The absence of odd-order terms in $\delta$ in Eq. (1) is due to the exchange symmetry between protons and neutrons in nuclear matter when one neglects the Coulomb interaction and assumes charge symmetry of nuclear forces. The nuclear symmetry energy $E_{\text {sym }}(\rho)$ thus corresponds to the lowest order coefficient. The higher order (including fourth-order) coefficients in $\delta$ are usually very small and neglected [e.g., the magnitude of the $\delta^{4}$ term at normal nuclear density $\rho_{0}$ (the saturation density of symmetric nuclear matter) is estimated to be less than $1 \mathrm{MeV}$ in microscopic many-body approaches [67-69] and also in phenomenological models as shown later]. Neglecting the contribution from higher order terms in Eq. (1) leads to the well-known empirical parabolic law for the EOS of asymmetric nuclear matter, which has been verified by all many-body theories to date, at least for densities up to moderate values [15]. As a good approximation, the density-dependent symmetry energy $E_{\text {sym }}(\rho)$ can thus be extracted from the parabolic approximation of $E_{\text {sym }}(\rho) \approx$ $E(\rho, \delta=1)-E(\rho, \delta=0)$. It should be mentioned that the possible presence of the higher order terms in $\delta$ at supranormal densities can significantly modify the proton fraction in $\beta$-equilibrium neutron-star matter and the critical density for the direct Urca process, which can lead to faster cooling of neutron stars [70,71]. In addition, a recent study [72,73] indicates that the higher order terms in $\delta$ are very important for determining the transition density and pressure at the inner edge separating the liquid core from the solid crust of neutron stars where the matter is extremely neutron rich.

Around the nuclear matter saturation density $\rho_{0}$, the binding energy per nucleon in symmetric nuclear matter $E_{0}(\rho)$ can be 
expanded (e.g., up to fourth-order in density) as

$$
E_{0}(\rho)=E_{0}\left(\rho_{0}\right)+L_{0} \chi+\frac{K_{0}}{2 !} \chi^{2}+\frac{J_{0}}{3 !} \chi^{3}+\frac{I_{0}}{4 !} \chi^{4}+O\left(\chi^{5}\right)
$$

where $\chi$ is a dimensionless variable characterizing the deviations of the density from the saturation density $\rho_{0}$ of symmetric nuclear matter and is conventionally defined as

$$
\chi=\frac{\rho-\rho_{0}}{3 \rho_{0}} .
$$

The first term $E_{0}\left(\rho_{0}\right)$ on the right-hand-side (r.h.s) of Eq. (4) is the binding energy per nucleon in symmetric nuclear matter at the saturation density $\rho_{0}$ and the coefficients of the other terms are

$$
\begin{aligned}
& L_{0}=\left.3 \rho_{0} \frac{d E_{0}(\rho)}{d \rho}\right|_{\rho=\rho_{0}}, \\
& K_{0}=\left.9 \rho_{0}^{2} \frac{d^{2} E_{0}(\rho)}{d \rho^{2}}\right|_{\rho=\rho_{0}}, \\
& J_{0}=\left.27 \rho_{0}^{3} \frac{d^{3} E_{0}(\rho)}{d \rho^{3}}\right|_{\rho=\rho_{0}}, \\
& I_{0}=\left.81 \rho_{0}^{4} \frac{d^{4} E_{0}(\rho)}{d \rho^{4}}\right|_{\rho=\rho_{0}} .
\end{aligned}
$$

Obviously, we have $L_{0}=0$ according to the definition of the saturation density $\rho_{0}$ of symmetric nuclear matter and thus the second term on the r.h.s of Eq. (4) should vanish. The coefficient $K_{0}$ is the so-called incompressibility coefficient of symmetric nuclear matter and it characterizes the curvature of $E_{0}(\rho)$ at $\rho_{0}$. The coefficients $J_{0}$ and $I_{0}$ correspond to higher order contributions and here we call them the third-order and fourth-order incompressibility coefficients of symmetric nuclear matter, respectively. In the literature, one usually neglects the higher order terms in Eq. (4) around the saturation density $\rho_{0}$ and obtains the following parabolic approximation to the EOS of symmetric nuclear matter:

$$
E_{0}(\rho)=E_{0}\left(\rho_{0}\right)+\frac{K_{0}}{2} \chi^{2}+O\left(\chi^{3}\right)
$$

Around the normal nuclear density $\rho_{0}$, the nuclear symmetry energy $E_{\text {sym }}(\rho)$ can be similarly expanded (e.g., up to fourth order in $\chi$ ) as

$$
\begin{aligned}
E_{\mathrm{sym}}(\rho)= & E_{\mathrm{sym}}\left(\rho_{0}\right)+L \chi+\frac{K_{\mathrm{sym}}}{2 !} \chi^{2} \\
& +\frac{J_{\mathrm{sym}}}{3 !} \chi^{3}+\frac{I_{\mathrm{sym}}}{4 !} \chi^{4}+O\left(\chi^{5}\right),
\end{aligned}
$$

where $L, K_{\text {sym }}, J_{\text {sym }}$, and $I_{\text {sym }}$ are the slope parameter, curvature parameter, third-order coefficient, and fourth-order coefficient of the nuclear symmetry energy at $\rho_{0}$, that is,

$$
\begin{aligned}
L & =\left.3 \rho_{0} \frac{d E_{\mathrm{sym}}(\rho)}{\partial \rho}\right|_{\rho=\rho_{0}}, \\
K_{\mathrm{sym}} & =\left.9 \rho_{0}^{2} \frac{d^{2} E_{\mathrm{sym}}(\rho)}{\partial \rho^{2}}\right|_{\rho=\rho_{0}},
\end{aligned}
$$

$$
\begin{aligned}
& J_{\text {sym }}=\left.27 \rho_{0}^{3} \frac{d^{3} E_{\text {sym }}(\rho)}{\partial \rho^{3}}\right|_{\rho=\rho_{0}}, \\
& I_{\text {sym }}=\left.81 \rho_{0}^{4} \frac{d^{4} E_{\text {sym }}(\rho)}{\partial \rho^{4}}\right|_{\rho=\rho_{0}} .
\end{aligned}
$$

The coefficients $L, K_{\text {sym }}, J_{\text {sym }}$, and $I_{\text {sym }}$ characterize the density dependence of the nuclear symmetry energy around the normal nuclear density $\rho_{0}$, and thus carry important information on the properties of nuclear symmetry energy at both high and low densities.

We can also expand the fourth-order nuclear symmetry energy $E_{\mathrm{sym}, 4}(\rho)$ around the normal nuclear density $\rho_{0}$ up to fourth order in $\chi$ as

$$
\begin{aligned}
E_{\mathrm{sym}, 4}(\rho)= & E_{\mathrm{sym}, 4}\left(\rho_{0}\right)+L_{\mathrm{sym}, 4} \chi+\frac{K_{\mathrm{sym}, 4}}{2} \chi^{2} \\
& +\frac{J_{\mathrm{sym}, 4}}{3 !} \chi^{3}+\frac{I_{\mathrm{sym}, 4}}{4 !} \chi^{4}+O\left(\chi^{5}\right),
\end{aligned}
$$

where $L_{\mathrm{sym}, 4}, K_{\mathrm{sym}, 4}, J_{\mathrm{sym}, 4}$, and $I_{\mathrm{sym}, 4}$ are the slope parameter, curvature parameter, third-order coefficient, and fourthorder coefficient of the fourth-order nuclear symmetry energy $E_{\text {sym }, 4}(\rho)$ at $\rho_{0}$, that is,

$$
\begin{aligned}
L_{\mathrm{sym}, 4} & =\left.3 \rho_{0} \frac{d E_{\mathrm{sym}, 4}(\rho)}{d \rho}\right|_{\rho=\rho_{0}}, \\
K_{\mathrm{sym}, 4} & =\left.9 \rho_{0}^{2} \frac{d^{2} E_{\mathrm{sym}, 4}(\rho)}{d \rho^{2}}\right|_{\rho=\rho_{0}}, \\
J_{\mathrm{sym}, 4} & =\left.27 \rho_{0}^{3} \frac{d^{3} E_{\mathrm{sym}, 4}(\rho)}{d \rho^{3}}\right|_{\rho=\rho_{0}}, \\
I_{\mathrm{sym}, 4} & =\left.81 \rho_{0}^{4} \frac{d^{4} E_{\mathrm{sym}, 4}(\rho)}{d \rho^{4}}\right|_{\rho=\rho_{0}} .
\end{aligned}
$$

In these Taylor expansions, we have kept all terms up to fourth order in $\delta$ or $\chi$. The 14 coefficients, namely, $E_{0}\left(\rho_{0}\right), K_{0}, J_{0}, I_{0}, E_{\text {sym }}\left(\rho_{0}\right), L, K_{\text {sym }}, J_{\text {sym }}, I_{\text {sym }}, E_{\text {sym }, 4}\left(\rho_{0}\right)$, $L_{\text {sym }, 4}, K_{\text {sym }, 4}, J_{\text {sym }, 4}$, and $I_{\text {sym }, 4}$, are well defined, and they characterize the EOS of asymmetric nuclear matter and its density dependence at normal nuclear density $\rho_{0}$. Among these parameters, $E_{0}\left(\rho_{0}\right), K_{0}, E_{\text {sym }}\left(\rho_{0}\right), L$, and $K_{\text {sym }}$ have been extensively studied in the literature and significant progress has been made over the past few decades. Based on these well-defined quantities, we investigate in the present paper to what extent they can provide reliable information on the isospin dependence of the saturation properties of asymmetric nuclear matter as well as its properties at both high and low densities.

\section{B. Saturation density}

One of the basic quantities for describing asymmetric nuclear matter is its saturation density $\rho_{\text {sat }}(\delta)$, which is generally a function of the isospin asymmetry $\delta$. According to the definition of the saturation density $\rho_{\text {sat }}(\delta)$ of asymmetric nuclear matter, that is,

$$
\left.\frac{\partial E(\rho, \delta)}{\partial \rho}\right|_{\rho=\rho_{\mathrm{sat}}(\delta)}=0
$$


it can be shown that up to fourth order in $\delta$, the saturation density can be expressed as (see the Appendix)

$$
\begin{aligned}
\rho_{\mathrm{sat}}(\delta)= & {\left[1-\frac{3 L}{K_{0}} \delta^{2}+\left(\frac{3 K_{\mathrm{sym}} L}{K_{0}^{2}}-\frac{3 L_{\mathrm{sym}, 4}}{K_{0}}-\frac{3 J_{0} L^{2}}{2 K_{0}^{3}}\right) \delta^{4}\right.} \\
& \left.+O\left(\delta^{6}\right)\right] \rho_{0} .
\end{aligned}
$$

Therefore, $\rho_{\text {sat }}(\delta)$ can be written as

$$
\rho_{\text {sat }}(\delta)=\rho_{0}+\rho_{\text {sat }, 2} \delta^{2}+\rho_{\text {sat }, 4} \delta^{4}+O\left(\delta^{6}\right),
$$

with

$$
\begin{aligned}
& \rho_{\text {sat }, 2}=-\frac{3 L}{K_{0}} \rho_{0}, \\
& \rho_{\text {sat }, 4}=\left(\frac{3 K_{\text {sym }} L}{K_{0}^{2}}-\frac{3 L_{\mathrm{sym}, 4}}{K_{0}}-\frac{3 J_{0} L^{2}}{2 K_{0}^{3}}\right) \rho_{0},
\end{aligned}
$$

which reflects the shift of the saturation density of asymmetric nuclear matter relative to that of symmetric nuclear matter owing to the finite isospin asymmetry.

\section{Binding energy at saturation density}

Another basic quantity of asymmetric nuclear matter is the binding energy per nucleon at saturation density [i.e., $\left.E_{\mathrm{sat}}(\delta)\right]$, which is generally a function of the isospin asymmetry $\delta$. According to Eq. (1) and Eq. (23), $E_{\text {sat }}(\delta)$ can be expressed up to fourth order in $\delta$ as

$$
\begin{aligned}
E_{\mathrm{sat}}(\delta)= & E_{0}\left(\rho_{0}\right)+\frac{K_{0}}{2 \times 9}\left(\frac{\rho_{\mathrm{sat}}-\rho_{0}}{\rho_{0}}\right)^{2} \\
& +\left[E_{\mathrm{sym}}\left(\rho_{0}\right)+\frac{L}{3}\left(\frac{\rho_{\mathrm{sat}}-\rho_{0}}{\rho_{0}}\right)\right] \delta^{2}+E_{\mathrm{sym}, 4}\left(\rho_{0}\right) \delta^{4} \\
= & E_{0}\left(\rho_{0}\right)+\frac{1}{2 \times 9} \frac{9 L^{2}}{K_{0}} \delta^{4}+E_{\mathrm{sym}}\left(\rho_{0}\right) \delta^{2} \\
& -\frac{L}{3} \frac{3 L}{K_{0}} \delta^{4}+E_{\mathrm{sym}, 4}\left(\rho_{0}\right) \delta^{4}+O\left(\delta^{6}\right) \\
= & E_{0}\left(\rho_{0}\right)+E_{\mathrm{sym}}\left(\rho_{0}\right) \delta^{2}+\left(E_{\mathrm{sym}, 4}\left(\rho_{0}\right)-\frac{L^{2}}{2 K_{0}}\right) \delta^{4} \\
& +O\left(\delta^{6}\right) .
\end{aligned}
$$

Therefore, $E_{\mathrm{sat}}(\delta)$ can be written as

$$
E_{\mathrm{sat}}(\delta)=E_{0}\left(\rho_{0}\right)+E_{\mathrm{sat}, 2} \delta^{2}+E_{\mathrm{sat}, 4} \delta^{4}+O\left(\delta^{6}\right),
$$

with

$$
\begin{aligned}
& E_{\text {sat }, 2}=E_{\text {sym }}\left(\rho_{0}\right), \\
& E_{\text {sat }, 4}=E_{\text {sym }, 4}\left(\rho_{0}\right)-\frac{L^{2}}{2 K_{0}} .
\end{aligned}
$$

The binding energy of asymmetric nuclear matter at saturation density is thus shifted from that of symmetry nuclear matter as a result of the shift of the saturation density when the isospin asymmetry is finite.

\section{Incompressibility at saturation density}

The incompressibility of asymmetric nuclear matter is an important quantity to describe its properties. It depends on the density and isospin asymmetry of the asymmetric nuclear matter and is characterized by the incompressibility coefficient defined as

$$
\begin{aligned}
K(\rho, \delta) & =9 \frac{\partial P(\rho, \delta)}{\partial \rho} \\
& =18 \rho \frac{\partial E(\rho, \delta)}{\partial \rho}+9 \rho^{2} \frac{\partial^{2} E(\rho, \delta)}{\partial \rho^{2}} \\
& =18 \frac{P(\rho, \delta)}{\rho}+9 \rho^{2} \frac{\partial^{2} E(\rho, \delta)}{\partial \rho^{2}},
\end{aligned}
$$

where $P(\rho, \delta)$ is the pressure of asymmetric nuclear matter, which can be expressed as

$$
P(\rho, \delta)=\rho^{2} \frac{\partial E(\rho, \delta)}{\partial \rho} .
$$

Conventionally, the incompressibility coefficient is defined at the saturation density where $P(\rho, \delta)=0$. It is also called the isobaric incompressibility coefficient [74] and is given by

$$
K_{\text {sat }}(\delta)=\left.9 \rho_{\text {sat }}^{2} \frac{\partial^{2} E(\rho, \delta)}{\partial \rho^{2}}\right|_{\rho=\rho_{\text {sat }}} .
$$

The isobaric incompressibility coefficient $K_{\text {sat }}(\delta)$ thus only depends on the isospin asymmetry $\delta$. One can show (see the Appendix) that, up to fourth order in $\delta$, the isobaric incompressibility coefficient $K_{\text {sat }}(\delta)$ can be expressed as

$$
\begin{aligned}
K_{\mathrm{sat}}(\delta)= & K_{0}+\left(K_{\mathrm{sym}}-6 L-\frac{J_{0}}{K_{0}} L\right) \delta^{2} \\
& +\left(K_{\mathrm{sym}, 4}-6 L_{\mathrm{sym}, 4}-\frac{J_{0} L_{\mathrm{sym}, 4}}{K_{0}}+\frac{9 L^{2}}{K_{0}}-\frac{J_{\mathrm{sym}} L}{K_{0}}\right. \\
& \left.+\frac{I_{0} L^{2}}{2 K_{0}^{2}}+\frac{J_{0} K_{\mathrm{sym}} L}{K_{0}^{2}}+\frac{3 J_{0} L^{2}}{K_{0}^{2}}-\frac{J_{0}^{2} L^{2}}{2 K_{0}^{3}}\right) \delta^{4} \\
& +O\left(\delta^{6}\right),
\end{aligned}
$$

which can be further written as

$$
K_{\mathrm{sat}}(\delta)=K_{0}+K_{\mathrm{sat}, 2} \delta^{2}+K_{\mathrm{sat}, 4} \delta^{4}+O\left(\delta^{6}\right),
$$

with

$$
\begin{aligned}
K_{\mathrm{sat}, 2}= & K_{\mathrm{sym}}-6 L-\frac{J_{0}}{K_{0}} L, \\
K_{\mathrm{sat}, 4}= & K_{\mathrm{sym}, 4}-6 L_{\mathrm{sym}, 4}-\frac{J_{0} L_{\mathrm{sym}, 4}}{K_{0}}+\frac{9 L^{2}}{K_{0}}-\frac{J_{\mathrm{sym}} L}{K_{0}} \\
& +\frac{I_{0} L^{2}}{2 K_{0}^{2}}+\frac{J_{0} K_{\mathrm{sym}} L}{K_{0}^{2}}+\frac{3 J_{0} L^{2}}{K_{0}^{2}}-\frac{J_{0}^{2} L^{2}}{2 K_{0}^{3}} .
\end{aligned}
$$

The coefficients $K_{\text {sat }, 2}$ and $K_{\text {sat, } 4}$ reflect the isospin dependence of the isobaric incompressibility of asymmetric nuclear matter.

If we use the parabolic approximation for the EOS of symmetric nuclear matter [i.e., Eq. (10)], then the $K_{\text {sat,2 }}$ parameter is reduced to

$$
K_{\text {asy }}=K_{\text {sym }}-6 L,
$$


and this expression has been extensively used in the literature to characterize the isospin dependence of the incompressibility of asymmetric nuclear matter [12,55,75-78]. Obviously, we have

$$
K_{\text {sat }, 2}=K_{\text {asy }}-\frac{J_{0}}{K_{0}} L
$$

and the coefficient $K_{\text {asy }}$ thus could be a good approximation to $K_{\text {sat, } 2}$ if the third-order derivative of the EOS of symmetric nuclear matter with respect to density (i.e., $J_{0}$ ), is negligible or the magnitude of the slope parameter $L$ for the symmetry energy is very small. It should be noted that the higher order derivatives of the EOS of symmetric nuclear matter with respect to density in Eq. (4), such as $I_{0}$, do not contribute

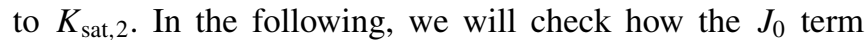
affects the value of $K_{\text {sat,2 }}$.

As shown in the Appendix, the expressions Eq. (23) for the saturation density, Eq. (27) for the binding energy, and Eq. (34) for the isobaric incompressibility coefficient are exact up to fourth order in $\delta$. It is thus interesting to see that, with precision up to fourth order in $\delta$, we only need to know 11 coefficients $\left[E_{0}\left(\rho_{0}\right), K_{0}, J_{0}, I_{0}, E_{\text {sym }}\left(\rho_{0}\right), L\right.$, $K_{\text {sym }}, J_{\text {sym }}, E_{\text {sym }, 4}\left(\rho_{0}\right), L_{\text {sym }, 4}$, and $\left.K_{\text {sym }, 4}\right]$ among the 14 coefficients $\left[E_{0}\left(\rho_{0}\right), K_{0}, J_{0}, I_{0}, E_{\text {sym }}\left(\rho_{0}\right), L, K_{\text {sym }}, J_{\text {sym }}, I_{\text {sym }}\right.$, $E_{\mathrm{sym}, 4}\left(\rho_{0}\right), L_{\mathrm{sym}, 4}, K_{\mathrm{sym}, 4}, J_{\mathrm{sym}, 4}$, and $\left.I_{\mathrm{sym}, 4}\right]$ that are defined at normal nuclear density $\rho_{0}$. The higher order coefficient $I_{\text {sym }}$ in Eq. (11) for the symmetry energy as well as $J_{\text {sym }, 4}$ and $I_{\mathrm{sym}, 4}$ in Eq. (16) for the fourth-order symmetry energy does not affect the saturation density, the binding energy, and the isobaric incompressibility coefficient with precision up to fourth order in $\delta$. Furthermore, the six coefficients $E_{0}\left(\rho_{0}\right), K_{0}, J_{0}, E_{\text {sym }}\left(\rho_{0}\right), L$, and $K_{\text {sym }}$ determine completely the saturation density, the binding energy, and the isobaric incompressibility coefficient with precision up to second order in $\delta$.

\section{THEORETICAL MODELS}

In this section, we introduce the theoretical models used in the present work and also give some important expressions for completeness. These models include the modified finite-range Gogny effective interaction (MDI) [43], the Hartree-Fock approach based on Skyrme interactions, and a phenomenological modified Skyrme-like (MSL) model. A very useful feature of these models is that analytical expressions for many interesting physical quantities in asymmetric nuclear matter at zero temperature can be obtained, and this makes it physically transparent and very convenient to check the higher order effects on the properties of asymmetric nuclear matter.

\section{A. Isospin- and momentum-dependent MDI interaction}

The MDI interaction is based on the finite-range Gogny effective interaction [43]. In the MDI interaction, the potential energy density $V(\rho, \delta)$ of asymmetric nuclear matter at total density $\rho$ and isospin asymmetry $\delta$ is expressed as $[43,55]$

$$
\begin{aligned}
V(\rho, \delta)= & \frac{A_{u} \rho_{n} \rho_{p}}{\rho_{0}}+\frac{A_{l}}{2 \rho_{0}}\left(\rho_{n}^{2}+\rho_{p}^{2}\right)+\frac{B}{\sigma+1} \frac{\rho^{\sigma+1}}{\rho_{0}^{\sigma}} \\
& \times\left(1-x \delta^{2}\right)+\frac{1}{\rho_{0}} \sum_{\tau, \tau^{\prime}} C_{\tau, \tau^{\prime}} \\
& \times \iint d^{3} p d^{3} p^{\prime} \frac{f_{\tau}(\vec{r}, \vec{p}) f_{\tau^{\prime}}\left(\vec{r}, \vec{p}^{\prime}\right)}{1+\left(\vec{p}-\vec{p}^{\prime}\right)^{2} / \Lambda^{2}},
\end{aligned}
$$

where $\tau=1 / 2(-1 / 2)$ for neutrons (protons), $\sigma=4 / 3$, and $f_{\tau}(\vec{r}, \vec{p})$ is the phase-space distribution function of neutrons or protons at coordinate $\vec{r}$ and momentum $\vec{p}$. The parameters $A_{u}(x), A_{l}(x), B, C_{\tau, \tau}, C_{\tau,-\tau}$, and $\Lambda$ are obtained by fitting the momentum dependence of the single-nucleon potential $U(\rho, \delta, \vec{p}, \tau)$ to that predicted by the Gogny Hartree-Fock and/or the Brueckner-Hartree-Fock calculations, the saturation properties of symmetric nuclear matter, and the symmetry energy at normal nuclear matter density $\rho_{0}$ [43]. The parameters $A_{u}(x)$ and $A_{l}(x)$ are given by

$$
\begin{aligned}
& A_{u}(x)=-95.98-x \frac{2 B}{\sigma+1}, \\
& A_{l}(x)=-120.57+x \frac{2 B}{\sigma+1}
\end{aligned}
$$

in terms of the parameter $x$, which is introduced to mimic various $E_{\mathrm{sym}}(\rho)$ predicted by different microscopic and/or phenomenological many-body theories [55]. By adjusting the $x$ parameter, we can vary $E_{\text {sym }}(\rho)$ without changing any property of symmetric nuclear matter and the symmetry energy at the saturation density, $E_{\text {sym }}\left(\rho_{0}\right)$, as the $x$-dependent $A_{u}(x)$ and $A_{l}(x)$ are adjusted accordingly. Using the definition in Eq. (2), we have $E_{\text {sym }}\left(\rho_{0}\right)=30.5 \mathrm{MeV}$ at $\rho_{0}=0.16 \mathrm{fm}^{-3}$ and its value is $31.6 \mathrm{MeV}$ within the parabolic approximation of $E_{\mathrm{sym}}(\rho) \approx$ $E(\rho, \delta=1)-E(\rho, \delta=0)$. We note that the MDI interaction has been extensively used in the transport model for studying isospin effects in intermediate-energy heavy-ion collisions induced by neutron-rich nuclei $[14,15,44,46,55,56,79-84]$, the study of the thermal properties of asymmetric nuclear matter [85-87], and the transition density and pressure in neutron star crust [72,73]. In particular, the isospin diffusion data from NSCL/MSU have constrained the value of $x$ to between 0 and -1 for nuclear densities from about $0.3 \rho_{0}$ to $1.2 \rho_{0}[55,56]$.

With $f_{\tau}(\vec{r}, \vec{p})=\frac{2}{h^{3}} \Theta\left[p_{f}(\tau)-p\right]$ for nuclear matter at zero temperature, the integrals in Eq. (39) can be evaluated analytically. In particular, we have [42]

$$
\begin{aligned}
\iint & d^{3} p d^{3} p^{\prime} \frac{f_{\tau}(\vec{r}, \vec{p}) f_{\tau^{\prime}}\left(\vec{r}, \vec{p}^{\prime}\right)}{1+\left(\vec{p}-\vec{p}^{\prime}\right)^{2} / \Lambda^{2}} \\
= & \frac{1}{6}\left(\frac{4 \pi}{h^{3}}\right)^{2} \Lambda^{2}\left\{p_{f}(\tau) p_{f}\left(\tau^{\prime}\right)\left[3\left(p_{f}^{2}(\tau)+p_{f}^{2}\left(\tau^{\prime}\right)\right)-\Lambda^{2}\right]\right. \\
& +4 \Lambda\left[\left(p_{f}^{3}(\tau)-p_{f}^{3}\left(\tau^{\prime}\right)\right) \arctan \frac{p_{f}(\tau)-p_{f}\left(\tau^{\prime}\right)}{\Lambda}\right. \\
& \left.-\left(p_{f}^{3}(\tau)+p_{f}^{3}\left(\tau^{\prime}\right)\right) \arctan \frac{p_{f}(\tau)+p_{f}\left(\tau^{\prime}\right)}{\Lambda}\right]
\end{aligned}
$$




$$
\begin{aligned}
& +\frac{1}{4}\left[\Lambda^{4}+6 \Lambda^{2}\left(p_{f}^{2}(\tau)+p_{f}^{2}\left(\tau^{\prime}\right)\right)\right. \\
& \left.\left.-3\left(p_{f}^{2}(\tau)-p_{f}^{2}\left(\tau^{\prime}\right)\right)^{2}\right] \ln \frac{\left(p_{f}(\tau)+p_{f}\left(\tau^{\prime}\right)\right)^{2}+\Lambda^{2}}{\left(p_{f}(\tau)-p_{f}\left(\tau^{\prime}\right)\right)^{2}+\Lambda^{2}}\right\} .
\end{aligned}
$$

The kinetic energy per nucleon of cold asymmetric nuclear matter is

$$
\begin{aligned}
E_{k}(\rho, \delta) & =\frac{1}{\rho} \int d^{3} p\left(\frac{p^{2}}{2 m} f_{n}(\vec{r}, \vec{p})+\frac{p^{2}}{2 m} f_{p}(\vec{r}, \vec{p})\right) \\
& =\frac{4 \pi}{5 m h^{3} \rho}\left(p_{f n}^{5}+p_{f p}^{5}\right),
\end{aligned}
$$

where $p_{f n(p)}=\hbar\left(3 \pi^{2} \rho_{n(p)}\right)^{1 / 3}$ is the Fermi momentum of neutrons (protons). The total energy per nucleon of cold asymmetric nuclear matter can be obtained as

$$
E(\rho, \delta)=\frac{V(\rho, \delta)}{\rho}+E_{k}(\rho, \delta) .
$$

By setting $\rho_{n}=\rho_{p}=\frac{\rho}{2}$ and $p_{f n}=p_{f p}=p_{f}$, where $p_{f}=$ $\hbar\left(3 \pi^{2} \rho / 2\right)^{1 / 3}$ is the fermi momentum of symmetric nuclear matter at density $\rho$, we obtain following EOS for cold symmetric nuclear matter:

$$
\begin{aligned}
E_{0}(\rho)= & \frac{8 \pi}{5 m h^{3} \rho} p_{f}^{5}+\frac{\rho}{4 \rho_{0}}\left(A_{l}+A_{u}\right)+\frac{B}{\sigma+1}\left(\frac{\rho}{\rho_{0}}\right)^{\sigma} \\
& +\frac{1}{3 \rho_{0} \rho}\left(C_{l}+C_{u}\right)\left(\frac{4 \pi}{h^{3}}\right)^{2} \Lambda^{2} \\
& \times\left\{p_{f}^{2}\left(6 p_{f}^{2}-\Lambda^{2}\right)-8 \Lambda p_{f}^{3} \arctan \frac{2 p_{f}}{\Lambda}\right. \\
& \left.+\frac{1}{4}\left[\Lambda^{4}+12 \Lambda^{2} p_{f}^{2}\right] \ln \frac{4 p_{f}^{2}+\Lambda^{2}}{\Lambda^{2}}\right\} .
\end{aligned}
$$

Furthermore, from Eq. (43) the symmetry energy can be expressed as

$$
\begin{aligned}
E_{\text {sym }}(\rho)= & \frac{1}{2}\left(\frac{\partial^{2} E}{\partial \delta^{2}}\right)_{\delta=0} \\
= & \frac{8 \pi}{9 m h^{3} \rho} p_{f}^{5}+\frac{\rho}{4 \rho_{0}}\left(A_{l}-A_{u}\right)-\frac{B x}{\sigma+1}\left(\frac{\rho}{\rho_{0}}\right)^{\sigma} \\
& +\frac{C_{l}}{9 \rho_{0} \rho}\left(\frac{4 \pi}{h^{3}}\right)^{2} \Lambda^{2}\left[4 p_{f}^{4}-\Lambda^{2} p_{f}^{2} \ln \frac{4 p_{f}^{2}+\Lambda^{2}}{\Lambda^{2}}\right] \\
& +\frac{C_{u}}{9 \rho_{0} \rho}\left(\frac{4 \pi}{h^{3}}\right)^{2} \Lambda^{2} \\
& \times\left[4 p_{f}^{4}-p_{f}^{2}\left(4 p_{f}^{2}+\Lambda^{2}\right) \ln \frac{4 p_{f}^{2}+\Lambda^{2}}{\Lambda^{2}}\right]
\end{aligned}
$$

and the fourth-order nuclear symmetry energy can be written as

$$
\begin{aligned}
E_{\mathrm{sym}, 4}(\rho)= & \frac{1}{4 !}\left(\frac{\partial^{4} E}{\partial \delta^{4}}\right)_{\delta=0}=\frac{8 \pi}{3^{5} m h^{3} \rho} p_{f}^{5} \\
& -\frac{C_{l}}{3^{5} \rho_{0} \rho}\left(\frac{4 \pi}{h^{3}}\right)^{2} \Lambda^{2}\left[7 \Lambda^{2} p_{f}^{2} \ln \frac{4 p_{f}^{2}+\Lambda^{2}}{\Lambda^{2}}\right.
\end{aligned}
$$

$$
\begin{aligned}
& \left.-\frac{4\left(7 \Lambda^{4} p_{f}^{4}+42 \Lambda^{2} p_{f}^{6}+40 p_{f}^{8}\right.}{\left(4 p_{f}^{2}+\Lambda^{2}\right)^{2}}\right] \\
& -\frac{C_{u}}{3^{5} \rho_{0} \rho}\left(\frac{4 \pi}{h^{3}}\right)^{2} \Lambda^{2}\left[\left(7 \Lambda^{2} p_{f}^{2}+16 p_{f}^{4}\right)\right. \\
& \left.\times \ln \frac{4 p_{f}^{2}+\Lambda^{2}}{\Lambda^{2}}-28 p_{f}^{4}-\frac{8 p_{f}^{6}}{\Lambda^{2}}\right] .
\end{aligned}
$$

\section{B. Skyrme-Hartree-Fock approach}

In the standard Skyrme Hartree-Fock model [64,88-96], the interaction is taken to have a zero-range, density- and momentum-dependent form with the interaction parameters determined from fitting the binding energies and charge radii of a large number of nuclei in the periodic table. In this approach, the EOS of asymmetric nuclear matter can be expressed as $[91,92,95,96]$

$$
\begin{aligned}
E(\rho, \delta)= & \frac{3 \hbar^{2}}{10 m}\left(\frac{3 \pi^{2}}{2}\right)^{2 / 3} \rho^{\frac{2}{3}} F_{5 / 3} \\
& +\frac{1}{8} t_{0} \rho\left[2\left(x_{0}+2\right)-\left(2 x_{0}+1\right) F_{2}\right] \\
& +\frac{1}{48} t_{3} \rho^{\sigma+1}\left[2\left(x_{3}+2\right)-\left(2 x_{3}+1\right) F_{2}\right] \\
& +\frac{3}{40}\left(\frac{3 \pi^{2}}{2}\right)^{2 / 3} \rho^{\frac{5}{3}}\left\{\left[t_{1}\left(x_{1}+2\right)+t_{2}\left(x_{2}+2\right)\right] F_{5 / 3}\right. \\
& \left.+\frac{1}{2}\left[t_{2}\left(2 x_{2}+1\right)-t_{1}\left(2 x_{1}+1\right)\right] F_{8 / 3}\right\},
\end{aligned}
$$

where

$$
F_{m}(\delta)=\frac{1}{2}\left[(1+\delta)^{m}+(1-\delta)^{m}\right] .
$$

The EOS of symmetric nuclear matter can thus be written as

$$
\begin{aligned}
E_{0}(\rho)= & \frac{3 \hbar^{2}}{10 m}\left(\frac{3 \pi^{2}}{2}\right)^{2 / 3} \rho^{\frac{2}{3}}+\frac{3}{8} t_{0} \rho \\
& +\frac{3}{80} \Theta_{s}\left(\frac{3 \pi^{2}}{2}\right)^{2 / 3} \rho^{\frac{5}{3}}+\frac{1}{16} t_{3} \rho^{\sigma+1},
\end{aligned}
$$

with $\Theta_{s}=3 t_{1}+\left(5+4 x_{2}\right) t_{2}$. Furthermore, the symmetry energy can be obtained as

$$
\begin{aligned}
E_{\mathrm{sym}}(\rho)= & \frac{1}{2}\left(\frac{\partial^{2} E}{\partial \delta^{2}}\right)_{\delta=0} \\
= & \frac{\hbar^{2}}{6 m}\left(\frac{3 \pi^{2}}{2}\right)^{2 / 3} \rho^{\frac{2}{3}}-\frac{1}{8} t_{0}\left(2 x_{0}+1\right) \rho \\
& -\frac{1}{24}\left(\frac{3 \pi^{2}}{2}\right)^{2 / 3} \Theta_{\mathrm{sym}} \rho^{\frac{5}{3}} \\
& -\frac{1}{48} t_{3}\left(2 x_{3}+1\right) \rho^{\sigma+1},
\end{aligned}
$$


with $\Theta_{\text {sym }}=3 t_{1} x_{1}-t_{2}\left(4+5 x_{2}\right)$. Similarly, the fourth-order nuclear symmetry energy can be written as

$$
\begin{aligned}
E_{\mathrm{sym}, 4}(\rho) & =\frac{1}{4 !}\left(\frac{\partial^{4} E}{\partial \delta^{4}}\right)_{\delta=0} \\
& =\frac{\hbar^{2}}{162 m}\left(\frac{3 \pi^{2}}{2}\right)^{2 / 3} \rho^{\frac{2}{3}}+\frac{1}{648}\left(\frac{3 \pi^{2}}{2}\right)^{2 / 3} \Theta_{\mathrm{sym}, 4} \rho^{\frac{5}{3}},
\end{aligned}
$$

with $\Theta_{\mathrm{sym}, 4}=t_{1}\left(1-x_{1}\right)+3 t_{2}\left(1+x_{2}\right)$.

\section{A phenomenological modified Skyrme-like model}

Following the energy density functional obtained from the Hartree-Fock approach with the zero-range, density- and momentum-dependent form with the Skyrme interaction, the binding energy per nucleon of cold asymmetric nuclear matter at total density $\rho$ and isospin asymmetry $\delta$ in the MSL model is parametrized as

$$
\begin{aligned}
E_{\mathrm{MSL}}(\rho, \delta)= & \frac{\eta}{\rho}\left(\frac{\hbar^{2}}{2 m_{n}^{*}} \rho_{n}^{5 / 3}+\frac{\hbar^{2}}{2 m_{p}^{*}} \rho_{p}^{5 / 3}\right) \\
& +\frac{\alpha}{2} \frac{\rho}{\rho_{0}}+\frac{\beta}{\sigma+1} \frac{\rho^{\sigma}}{\rho_{0}^{\sigma}}+E_{\mathrm{sym}}^{\mathrm{loc}}(\rho) \delta^{2},
\end{aligned}
$$

where $\eta=\frac{3}{5}\left(3 \pi^{2}\right)^{2 / 3} ; \alpha, \beta$, and $\sigma$ are parameters; and $E_{\text {sym }}^{\text {loc }}(\rho)$ represents the local density-dependent part of the symmetry energy. The effective neutron and proton masses $m_{n}^{*}$ and $m_{p}^{*}$ are assumed to have forms similar to the standard SHF results $[91,92,95,96]$, that is,

$$
\begin{aligned}
\frac{\hbar^{2}}{2 m_{n}^{*}} & =\frac{\hbar^{2}}{2 m}+\rho\left(C_{\mathrm{eff}}+D_{\mathrm{eff}} \delta\right), \\
\frac{\hbar^{2}}{2 m_{p}^{*}} & =\frac{\hbar^{2}}{2 m}+\rho\left(C_{\mathrm{eff}}-D_{\mathrm{eff}} \delta\right),
\end{aligned}
$$

where $C_{\text {eff }}$ and $D_{\text {eff }}$ are constants. This implies that the single-nucleon potential depends quadratically on the nucleon momentum as in the standard SHF approach. In terms of the isoscalar effective mass $m_{s}^{*}$ and the isovector effective mass $m_{v}^{*}$ given by

$$
\begin{aligned}
\frac{\hbar^{2}}{2 m_{s}^{*}} & =\frac{\hbar^{2}}{2 m}+\rho C_{\mathrm{eff}}, \\
\frac{\hbar^{2}}{2 m_{v}^{*}} & =\frac{\hbar^{2}}{2 m}+\rho\left(C_{\mathrm{eff}}-D_{\mathrm{eff}}\right),
\end{aligned}
$$

the nucleon effective mass can be written as [97]

$$
\frac{\hbar^{2}}{2 m_{q}^{*}}=\frac{2 \rho_{q}}{\rho} \frac{\hbar^{2}}{2 m_{s}^{*}}+\left(1-\frac{2 \rho_{q}}{\rho}\right) \frac{\hbar^{2}}{2 m_{v}^{*}}, \quad q=n, p .
$$

We note that the isovector effective mass $m_{v}^{*}$ corresponds to the proton (neutron) effective mass in pure neutron (proton) matter. Also, we can easily obtain the following relation:

$$
\frac{\hbar^{2}}{2 m_{n}^{*}}-\frac{\hbar^{2}}{2 m_{p}^{*}}=2 \delta\left(\frac{\hbar^{2}}{2 m_{s}^{*}}-\frac{\hbar^{2}}{2 m_{v}^{*}}\right) .
$$

Experimentally, the isoscalar effective mass $m_{s}^{*}$ and the isovector effective mass $m_{v}^{*}$ at normal nuclear density $\rho_{0}$ have been constrained to be $m_{s, 0}^{*} \approx 0.8 m$ and $m_{v, 0}^{*} \approx 0.7 m$, respectively $[42,91,92,98-103]$. With these constrained values for the isoscalar and isovector effective masses, Eq. (56) gives a larger neutron effective mass than the proton effective mass in neutron-rich matter, which is consistent with experimental data on the isospin dependence of the nucleon optical potential and also with recent microscopic and phenomenological manybody theory predictions $[15,33,45]$.

The EOS of symmetric nuclear matter in the MSL model is then given by

$$
\begin{aligned}
E_{0}(\rho)= & E_{\mathrm{kin}}^{0}\left(\frac{\rho}{\rho_{0}}\right)^{2 / 3}+C\left(\frac{\rho}{\rho_{0}}\right)^{5 / 3} \\
& +\frac{\alpha}{2} \frac{\rho}{\rho_{0}}+\frac{\beta}{\sigma+1}\left(\frac{\rho}{\rho_{0}}\right)^{\sigma},
\end{aligned}
$$

where the first term represents the kinetic energy contribution with $E_{\mathrm{kin}}^{0}=\frac{3 \hbar^{2}}{10 m}\left(\frac{3 \pi^{2}}{2}\right)^{2 / 3} \rho_{0}^{2 / 3}$ and the second term is due to the nucleon effective mass with the coefficient $C$ being a constant determined by the isoscalar effective mass $m_{s, 0}^{*}$ as

$$
C=\frac{m-m_{s, 0}^{*}}{m_{s, 0}^{*}} E_{\mathrm{kin}}^{0} .
$$

The parameters $\alpha, \beta$, and $\sigma$ in the MSL model are determined by the binding energy per nucleon $E_{0}\left(\rho_{0}\right)$ and the incompressibility $K_{0}$ of cold symmetric nuclear matter at the saturation density $\rho_{0}$, and they can be expressed as

$$
\begin{aligned}
\alpha= & -\frac{4}{3} E_{\mathrm{kin}}^{0}-\frac{10}{3} C-\frac{2}{3}\left[E_{\mathrm{kin}}^{0}-3 E_{0}\left(\rho_{0}\right)-2 C\right] \\
& \times \frac{K_{0}+2 E_{\mathrm{kin}}^{0}-10 C}{K_{0}+9 E_{0}\left(\rho_{0}\right)-E_{\mathrm{kin}}^{0}-4 C}, \\
\beta= & {\left[\frac{\left.E_{\mathrm{kin}}^{0}-E_{0}\left(\rho_{0}\right)-\frac{2}{3} C\right]}{3}\right.} \\
& \times \frac{K_{0}-9 E_{0}\left(\rho_{0}\right)+5 E_{\mathrm{kin}}^{0}-16 C}{K_{0}+9 E_{0}\left(\rho_{0}\right)-E_{\mathrm{kin}}^{0}-4 C}, \\
\sigma= & \frac{K_{0}+2 E_{\mathrm{kin}}^{0}-10 C}{3 E_{\mathrm{kin}}^{0}-9 E_{0}\left(\rho_{0}\right)-6 C} .
\end{aligned}
$$

In particular, for $E_{0}\left(\rho_{0}\right)=-16 \mathrm{MeV}, m_{s, 0}^{*}=0.8 m$, and $\rho_{0}=$ $0.16 \mathrm{fm}^{-3}$, we have

$$
\begin{aligned}
C & =5.53(\mathrm{MeV}), \\
\alpha & =-47.90-39.37 \frac{K_{0}-11.05}{K_{0}-188.21}(\mathrm{MeV}), \\
\beta & =19.68 \frac{K_{0}+166.11}{K_{0}-188.21}(\mathrm{MeV}), \\
\sigma & =\frac{K_{0}-11.05}{177.16},
\end{aligned}
$$

where the units of $K_{0}$ are $\mathrm{MeV}$.

The symmetry energy in the MSL model can be expressed as

$E_{\mathrm{sym}}(\rho)=E_{\mathrm{sym}}^{\mathrm{kin}}\left(\rho_{0}\right)\left(\frac{\rho}{\rho_{0}}\right)^{2 / 3}+D\left(\frac{\rho}{\rho_{0}}\right)^{5 / 3}+E_{\mathrm{sym}}^{\mathrm{loc}}(\rho)$, 
where the first term is the kinetic energy contribution with $E_{\mathrm{sym}}^{\mathrm{kin}}\left(\rho_{0}\right)=\frac{\hbar^{2}}{6 m}\left(\frac{3 \pi^{2}}{2} \rho_{0}\right)^{2 / 3}$ and the second term is due to the contribution of the nucleon effective mass with the coefficient $D$ determined by both $m_{s, 0}^{*}$ and $m_{v, 0}^{*}$ as

$$
D=\frac{5}{9} E_{\text {kin }}^{0}\left(4 \frac{m}{m_{s, 0}^{*}}-3 \frac{m}{m_{v, 0}^{*}}-1\right)
$$

With $m_{s, 0}^{*}=0.8 m$ and $m_{v, 0}^{*}=0.7 m$ at $\rho_{0}=0.16 \mathrm{fm}^{-3}$, we have $D=-3.51 \mathrm{MeV}$. In Eq. (66), similarly to the momentum-independent (MID) model [51], the local densitydependent part $E_{\text {sym }}^{\text {loc }}(\rho)$ is parametrized as

$E_{\mathrm{sym}}^{\mathrm{loc}}(\rho)=(1-y) E_{\mathrm{sym}}^{\mathrm{loc}}\left(\rho_{0}\right) \frac{\rho}{\rho_{0}}+y E_{\mathrm{sym}}^{\mathrm{loc}}\left(\rho_{0}\right)\left(\frac{\rho}{\rho_{0}}\right)^{\gamma_{\mathrm{sym}}}$,

with the constant $E_{\mathrm{sym}}^{\mathrm{loc}}\left(\rho_{0}\right)$ determined by

$$
E_{\mathrm{sym}}^{\mathrm{loc}}\left(\rho_{0}\right)=E_{\mathrm{sym}}\left(\rho_{0}\right)-E_{\mathrm{sym}}^{\mathrm{kin}}\left(\rho_{0}\right)-D .
$$

Obviously, we have $E_{\mathrm{sym}}^{\mathrm{loc}}\left(\rho_{0}\right)=21.2 \mathrm{MeV}$ following $E_{\text {sym }}\left(\rho_{0}\right)=30 \mathrm{MeV}$ and $E_{\text {sym }}^{\text {kin }}\left(\rho_{0}\right)=12.3 \mathrm{MeV}$ at $\rho_{0}=$ $0.16 \mathrm{fm}^{-3}$. The default value for the $\gamma_{\text {sym }}$ parameter is taken to be $4 / 3$ in the MSL model following the energy $E_{\text {sym }}(\rho)$ in the MDI interaction, namely, Eq. (45). (We will see how the $\gamma_{\text {sym }}$ parameter affects the symmetry energy in the following.) In particular, similarly to the $x$ parameter in the MDI interaction, the dimensionless parameter $y$ in the MSL model is introduced to mimic various $E_{\text {sym }}(\rho)$ values predicted by different microscopic and/or phenomenological many-body theories for a fixed $\gamma_{\text {sym }}$ parameter. As we will show later, for $\gamma_{\text {sym }}=4 / 3$, adjusting the $y$ value can nicely reproduce the symmetry energy $E_{\text {sym }}(\rho)$ in the MDI interaction with $x=-1,0$, and 1 . Therefore, with the variation of only one parameter, the symmetry energy density functional constructed in the MSL model is very flexible and can mimic very different density behaviors.

In the MSL model, similarly to the SHF approach, the fourth-order and higher order nuclear symmetry energies only include contributions from the kinetic energy and the nucleon effective mass whereas the local density-dependent part of higher order symmetry energies is neglected in Eq. (50). In particular, the fourth-order nuclear symmetry energy in the MSL model can be shown to be

$$
\begin{aligned}
E_{\mathrm{sym}, 4}(\rho) & =\frac{1}{4 !}\left(\frac{\partial^{4} E}{\partial \delta^{4}}\right)_{\delta=0} \\
& =\frac{5}{243} E_{\mathrm{kin}}^{0}\left(\frac{\rho}{\rho_{0}}\right)^{2 / 3}+\frac{5}{243} C\left(\frac{\rho}{\rho_{0}}\right)^{5 / 3},
\end{aligned}
$$

where the first term is the kinetic energy contribution and the second term is due to the contribution from the nucleon effective mass.

The MSL model is thus an extension of the MID model [51] by including the effects of the nucleon effective mass. It provides a simple phenomenological parametrization of the EOS of asymmetric nuclear matter and is thus a convenient and transparent way to investigate the possible correlations among higher order and lower order characteristic parameters of asymmetric nuclear matter. In the MSL model, we have a total of eight free parameters [i.e., $C, D, \alpha, \beta, \sigma, E_{\text {sym }}\left(\rho_{0}\right), y$, and $\gamma_{\text {sym }}$ ], which can be determined by empirical information on the EOS of symmetric nuclear matter, the nucleon effective mass, and the density dependence of symmetry energy. In particular, the parameters $C$ and $D$ (or equivalently $C_{\text {eff }}$ and $D_{\text {eff }}$ ) are determined by the isoscalar effective masses $m_{s}^{*}$ and the isovector effective mass $m_{v}^{*}$ at normal nuclear density $\rho_{0}$ (i.e., $m_{s, 0}^{*}$ and $m_{v, 0}^{*}$ ). The parameters $\alpha, \beta$, and $\sigma$ are determined by $E_{0}\left(\rho_{0}\right), K_{0}$, and $\rho_{0}$ and the parameters $E_{\text {sym }}\left(\rho_{0}\right), y$, and $\gamma_{\text {sym }}$ are introduced to mimic the density dependence of different symmetry energies predicted by microscopic and/or phenomenological many-body theories. As a default in the MSL model, we use $m_{s, 0}^{*}=0.8 m, m_{v, 0}^{*}=0.7 m, \rho_{0}=$ $0.16 \mathrm{fm}^{-3}, E_{0}\left(\rho_{0}\right)=-16 \mathrm{MeV}, K_{0}=240 \mathrm{MeV}, E_{\mathrm{sym}}\left(\rho_{0}\right)=$ $30 \mathrm{MeV}$, and $\gamma_{\text {sym }}=4 / 3$ and vary the parameter $y$ to describe different symmetry energies.

\section{RESULTS AND DISCUSSION}

\section{A. Characteristic parameters at normal nuclear density and EOS of asymmetric nuclear matter}

As shown in Sec. II, the expressions for the saturation density, Eq. (23), the binding energy, Eq. (27), and the isobaric incompressibility coefficient, Eq. (34), are exact up to fourth order in $\delta$, and these expressions involve 11 characteristic parameters defined at the normal nuclear density $\rho_{0}$ [i.e., $E_{0}\left(\rho_{0}\right), K_{0}, J_{0}, I_{0}, E_{\text {sym }}\left(\rho_{0}\right), L, K_{\text {sym }}, J_{\text {sym }}$, $E_{\text {sym }, 4}\left(\rho_{0}\right), L_{\text {sym }, 4}$, and $\left.K_{\text {sym }, 4}\right]$. We summarize the values of $\rho_{0}, E_{0}\left(\rho_{0}\right), K_{0}, J_{0}, I_{0}, K_{\text {asy }}, K_{\text {sat }, 2}$, and $K_{\text {sat }, 2}$ in Table I and the values of $E_{\text {sym }}\left(\rho_{0}\right), L, K_{\text {sym }}, J_{\text {sym }}, E_{\text {sym }, 4}\left(\rho_{0}\right), L_{\text {sym }, 4}, K_{\text {sym }, 4}$, $m_{s, 0}^{*} / m$, and $m_{v, 0}^{*} / m$ in Table II for the MDI interaction with $x=1,0$, and -1 and the popular 63 standard Skyrme interactions with their saturation density and the symmetry energy satisfying $0.140<\rho_{0}<0.165 \mathrm{fm}^{-3}$ and $25<E_{\text {sym }}\left(\rho_{0}\right)<$ $37 \mathrm{MeV}$, respectively. For the 63 standard Skyrme interactions, the values in the tables are sorted in the order of increasing values of $L$. It should be stressed here that the parameters of all Skyrme interactions are chosen to fit the binding energies and charge radii of a large number of nuclei in the periodic table. Detailed values of the parameters for these 63 Skyrme interactions can be found in Refs. [50,89-93,95,96,104-113]. The selected ranges of $\rho_{0}$ and $E_{\text {sym }}\left(\rho_{0}\right)$ are essentially consistent with their empirical values inferred from experimental data. We note that here no constraints are imposed on $K_{0}$ and $L$ for selecting the Skyrme interactions as we will systematically explore the correlations of other physical quantities with $K_{0}$ or $L$.

Since the 11 characteristic parameters are defined at the normal nuclear density $\rho_{0}$, it is of interest to study the extent to which these characteristic parameters can provide information on the properties of asymmetric nuclear at subsaturation and supra-saturation density regions. As an example, we show in Fig. 1 the energy per nucleon of symmetric nuclear matter from the MDI interaction as a function of its density. Also shown in Fig. 1 are the results obtained by using Eq. (4) including terms up to $\chi^{2}, \chi^{3}$, and $\chi^{4}$, respectively. It is seen that Eq. (4) with terms up to $\chi^{2}$ [i.e., the parabolic approximation Eq. (10), which involves only two characteristic parameters- $E_{0}\left(\rho_{0}\right)$ 
TABLE I. The saturation density $\rho_{0}$ and the characteristic parameters $E_{0}\left(\rho_{0}\right)(\mathrm{MeV}), K_{0}(\mathrm{MeV}), J_{0}(\mathrm{MeV})$, $I_{0}(\mathrm{MeV}), K_{\text {asy }}(\mathrm{MeV}), K_{\text {sat }, 2}(\mathrm{MeV})$, and $K_{\text {sat }, 4}(\mathrm{MeV})$ at saturation density for the MDI interaction with $x=-1,0$, and 1 and the SHF predictions with 63 standard Skyrme interactions. The small differences from Table I of Ref. [73] for some Skyrme interactions are due to the use of $0.17(0.33)$ as an approximation of $1 / 6(1 / 3)$ for the $\sigma$ parameter in Ref. [73]. The results shown here are thus more accurate than those in Ref. [73].

\begin{tabular}{|c|c|c|c|c|c|c|c|c|}
\hline Force & $\rho_{0}$ & $E_{0}\left(\rho_{0}\right)$ & $K_{0}$ & $J_{0}$ & $I_{0}$ & $K_{\text {asy }}$ & $K_{\text {sat }, 2}$ & $K_{\text {sat }, 4}$ \\
\hline MDI(1) & 0.160 & -16.2 & 212.5 & -447.6 & 2160.8 & -352.0 & -321.1 & -8.4 \\
\hline $\operatorname{MDI}(0)$ & 0.160 & -16.2 & 212.5 & -447.6 & 2160.8 & -443.1 & -316.3 & 52.9 \\
\hline $\operatorname{MDI}(-1)$ & 0.160 & -16.2 & 212.5 & -447.6 & 2160.8 & -534.3 & -311.4 & 214.4 \\
\hline$Z$ & 0.159 & -16.0 & 330.3 & -65.0 & -348.2 & -359.6 & -369.4 & 100.5 \\
\hline$E_{\sigma}$ & 0.163 & -16.0 & 248.6 & -352.4 & 1337.1 & -236.6 & -288.9 & 57.9 \\
\hline$E$ & 0.159 & -16.1 & 333.5 & -63.0 & -356.3 & -383.1 & -389.1 & 41.1 \\
\hline$Z_{\sigma}$ & 0.163 & -15.9 & 233.3 & -369.0 & 1546.0 & -225.1 & -271.6 & 43.3 \\
\hline SVI & 0.143 & -15.8 & 363.6 & 153.5 & -1107.4 & -427.3 & -424.2 & -4.4 \\
\hline$Z_{\sigma}^{*}$ & 0.162 & -16.0 & 234.9 & -369.2 & 1544.4 & -305.5 & -312.6 & -7.1 \\
\hline SkSC4 & 0.161 & -15.9 & 234.7 & -380.8 & 1549.2 & -316.5 & -320.1 & -4.1 \\
\hline SI & 0.155 & -16.0 & 370.4 & 152.3 & -1129.5 & -469.2 & -469.7 & -14.2 \\
\hline BSk3 & 0.157 & -15.8 & 234.8 & -380.9 & 1529.8 & -347.6 & -336.6 & -9.2 \\
\hline BSk1 & 0.157 & -15.8 & 231.3 & -385.6 & 1588.7 & -325.0 & -313 & -8.4 \\
\hline SIII & 0.145 & -15.9 & 355.4 & 101.4 & -903.0 & -453.2 & -456.0 & -20.2 \\
\hline BSk2 & 0.157 & -15.8 & 233.7 & -380.1 & 1542.4 & -344.8 & -331.9 & -9.7 \\
\hline MSk7 & 0.157 & -15.8 & 231.2 & -385.4 & 1587.3 & -331.0 & -315.4 & -8.6 \\
\hline BSk4 & 0.157 & -15.8 & 236.8 & -367.2 & 1466.9 & -341.2 & -321.7 & -11.4 \\
\hline BSk8 & 0.159 & -15.8 & 230.3 & -372.4 & 1578.2 & -310.0 & -286.0 & -16.8 \\
\hline BSk6 & 0.157 & -15.8 & 229.1 & -370.6 & 1571.3 & -316.3 & -289.0 & -16.8 \\
\hline BSk7 & 0.157 & -15.8 & 229.3 & -370.9 & 1572.8 & -317.3 & -288.2 & -17.0 \\
\hline SKP & 0.163 & -16.0 & 201.0 & -435.6 & 2127.8 & -384.3 & -341.9 & 7.4 \\
\hline BSk5 & 0.157 & -15.8 & 237.2 & -367.9 & 1470.3 & -368.8 & -335.6 & -7.5 \\
\hline SKXm & 0.159 & -16.0 & 238.1 & -380.4 & 1542.2 & -435.3 & -384.0 & 10.3 \\
\hline RATP & 0.160 & -16.0 & 239.4 & -349.7 & 1451.5 & -385.5 & -338.2 & -6.6 \\
\hline SKX & 0.155 & -16.1 & 271.1 & -297.4 & 904.0 & -451.2 & -414.8 & 2.3 \\
\hline SKXce & 0.156 & -15.9 & 268.2 & -294.6 & 892.9 & -439.3 & -402.5 & 3.8 \\
\hline BSk15 & 0.159 & -16.0 & 241.6 & -363.1 & 1457.0 & -395.9 & -345.4 & -2.7 \\
\hline BSk16 & 0.159 & -16.1 & 241.7 & -363.6 & 1459.9 & -396.6 & -344.2 & -1.7 \\
\hline BSk10 & 0.159 & -15.9 & 238.8 & -370.3 & 1479.6 & -418.3 & -360.6 & 12.7 \\
\hline SGII & 0.158 & -15.6 & 214.7 & -380.9 & 1741.8 & -371.7 & -304.9 & 17.8 \\
\hline BSk12 & 0.159 & -15.9 & 238.1 & -369.1 & 1474.9 & -419.4 & -360.5 & 14.0 \\
\hline BSk11 & 0.159 & -15.9 & 238.1 & -369.2 & 1475.3 & -420.0 & -360.5 & 14.6 \\
\hline SLy10 & 0.156 & -15.9 & 229.7 & -358.3 & 1559.6 & -374.7 & -314.2 & -24.6 \\
\hline BSk13 & 0.159 & -15.9 & 238.1 & -369.2 & 1475.2 & -420.8 & -360.6 & 15.5 \\
\hline BSk9 & 0.159 & -15.9 & 231.4 & -374.9 & 1591.5 & -384.7 & -320.0 & -3.1 \\
\hline BSk14 & 0.159 & -15.9 & 239.3 & -358.7 & 1434.8 & -415.5 & -349.7 & 14.2 \\
\hline SLy230a & 0.160 & -16.0 & 229.9 & -364.2 & 1593.6 & -364.1 & -293.9 & -32.1 \\
\hline SLy6 & 0.159 & -15.9 & 229.9 & -360.2 & 1568.8 & -383.7 & -312.9 & -13.2 \\
\hline SLy8 & 0.160 & -16.0 & 229.9 & -363.2 & 1587.1 & -388.4 & -316.8 & -12.0 \\
\hline SLy4 & 0.160 & -16.0 & 229.9 & -363.1 & 1586.9 & -392.1 & -320.5 & -12.7 \\
\hline SLy0 & 0.161 & -16.0 & 230.2 & -365.2 & 1598.7 & -389.2 & -317.2 & -11.6 \\
\hline SLy3 & 0.160 & -16.0 & 229.9 & -363.4 & 1588.0 & -395.4 & -323.4 & -12.9 \\
\hline SKM* & 0.160 & -15.8 & 216.6 & -386.1 & 1768.9 & -430.6 & -349.0 & 37.3 \\
\hline SLy230b & 0.160 & -16.0 & 229.9 & -363.1 & 1586.8 & -395.5 & -322.9 & -12.2 \\
\hline SLy7 & 0.158 & -15.9 & 229.7 & -359.2 & 1562.9 & -402.7 & -327.6 & -10.4 \\
\hline SLy2 & 0.160 & -15.9 & 229.2 & -362.7 & 1585.5 & -406.1 & -328.9 & -7.0 \\
\hline SLy1 & 0.160 & -16.0 & 229.8 & -364.3 & 1594.4 & -408.7 & -331.3 & -7.3 \\
\hline SKM & 0.160 & -15.8 & 216.6 & -386.1 & 1768.9 & -444.9 & -356.9 & 45.1 \\
\hline SII & 0.148 & -16.0 & 341.4 & 15.8 & -567.5 & -565.9 & -568.2 & 23.2 \\
\hline SLy5 & 0.161 & -16.0 & 229.9 & -364.1 & 1592.7 & -413.7 & -334.0 & -4.6 \\
\hline SLy9 & 0.151 & -15.8 & 229.8 & -350.4 & 1511.4 & -413.7 & -329.2 & 1.7 \\
\hline SkI6 & 0.159 & -15.9 & 248.2 & -326.6 & 1251.8 & -402.2 & -324.3 & 15.5 \\
\hline SkI4 & 0.160 & -15.9 & 248.0 & -331.2 & 1280.0 & -402.9 & -322.2 & 21.9 \\
\hline
\end{tabular}


TABLE I. (Continued.)

\begin{tabular}{lcccccrrr}
\hline \hline Force & $\rho_{0}$ & $E_{0}\left(\rho_{0}\right)$ & $K_{0}$ & $J_{0}$ & $I_{0}$ & $K_{\text {asy }}$ & $K_{\text {sat }, 2}$ & $K_{\text {sat }, 4}$ \\
\hline SIV & 0.151 & -16.0 & 324.6 & -68.8 & -234.9 & -517.7 & -504.2 & 64.8 \\
SGI & 0.154 & -15.9 & 261.8 & -297.9 & 1005.0 & -435.2 & -362.5 & 61.8 \\
SKO* & 0.160 & -15.7 & 222.1 & -390.2 & 1706.7 & -495.6 & -373.2 & 98.3 \\
SkMP & 0.157 & -15.6 & 231.0 & -338.4 & 1425.2 & -468.9 & -366.8 & 101.5 \\
SKa & 0.155 & -16.0 & 263.2 & -300.1 & 1014.4 & -526.2 & -441.1 & 105.0 \\
SKO & 0.160 & -15.8 & 222.8 & -391.3 & 1712.3 & -519.1 & -379.5 & 143.9 \\
$R_{\sigma}$ & 0.158 & -15.6 & 237.4 & -348.4 & 1377.2 & -523.3 & -397.5 & 184.5 \\
$G_{\sigma}$ & 0.158 & -15.6 & 237.2 & -348.7 & 1379.5 & -550.1 & -411.9 & 230.6 \\
SKT4 & 0.159 & -16.0 & 235.5 & -383.0 & 1562.5 & -589.3 & -436.2 & 201.2 \\
SV & 0.155 & -16.0 & 305.7 & -175.8 & 183.5 & -552.4 & -497.1 & 176.2 \\
SkI3 & 0.158 & -16.0 & 258.2 & -303.9 & 1088.3 & -530.1 & -411.8 & 152.1 \\
SkI2 & 0.158 & -15.8 & 240.9 & -339.7 & 1351.4 & -555.3 & -408.2 & 226.9 \\
SkI5 & 0.156 & -15.8 & 255.8 & -302.0 & 1083.7 & -616.4 & -463.7 & 347.2 \\
\hline \hline
\end{tabular}

and $K_{0}$ ] can approximate very well the EOS of symmetric nuclear matter from about $0.5 \rho_{0}$ to $1.5 \rho_{0}$. Including higher order terms of $\chi^{3}$ and $\chi^{4}$ with the characteristic parameters $J_{0}$ and $I_{0}$ in Eq. (4) significantly improves the approximation to the EOS at low densities and that up to about $2 \rho_{0}$. To describe reasonably the EOS of symmetric nuclear matter above $2 \rho_{0}$, one needs to include higher order terms in $\chi$.

Figure 2 displays the density dependence of symmetry energy using the MDI interaction with $x=1,0$, and -1 together with corresponding results obtained by using Eq. (11) up to $\chi, \chi^{2}, \chi^{3}$, and $\chi^{4}$, respectively. It is seen that the importance of the contributions from higher order terms in $\chi$ to the density dependence of the symmetry energy depends on the stiffness of symmetry energy. For a supra-soft symmetry energy $(x=1)$, terms up to $\chi^{3}$ are needed to describe reasonably the symmetry energy from subsaturation densities to about $2 \rho_{0}$. The situation is similar for the case of modestly soft symmetry energy $(x=0)$. For the stiffer symmetry energy $(x=-1)$, including terms up to $\chi^{2}$ already give a

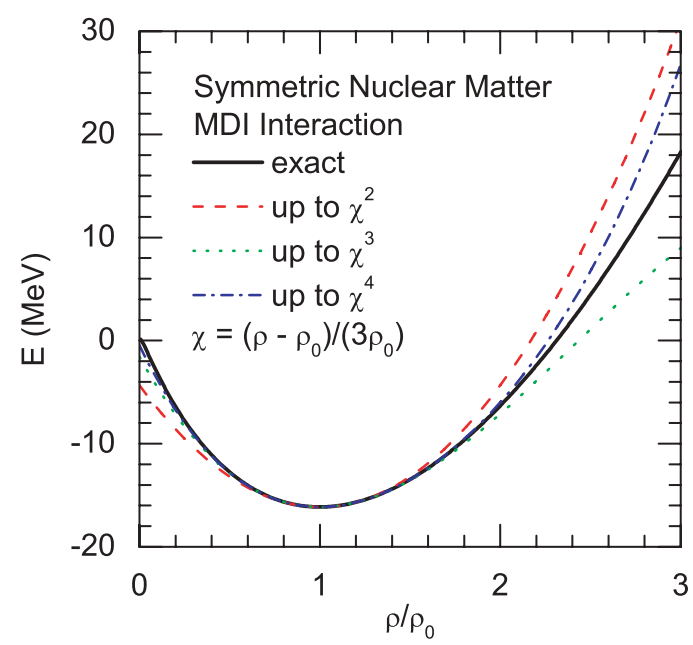

FIG. 1. (Color online) Energy per nucleon as a function of density for symmetric nuclear matter in the MDI interaction. Also included are results obtained by using Eq. (4) up to $\chi^{2}, \chi^{3}$, and $\chi^{4}$, respectively. good description of the symmetry energy from subsaturation densities to about $3 \rho_{0}$.

In Fig. 3, we show the density dependence of the fourthorder symmetry energy $E_{\text {sym }, 4}(\rho)$ using the MDI interaction together with corresponding results obtained by using Eq. (16) up to $\chi, \chi^{2}, \chi^{3}$, and $\chi^{4}$, respectively. We note here that $E_{\text {sym }, 4}(\rho)$ does not depend on the $x$ parameter in the MDI interaction as shown in Eq. (46). First, one can see from Fig. 3 that the magnitude of $E_{\mathrm{sym}, 4}(\rho)$ is very small compared to that of $E_{\text {sym }}(\rho)$. As shown in Table II, the value of $E_{\text {sym }, 4}(\rho)$ at normal nuclear density $\rho_{0}$ is about $0.62 \mathrm{MeV}$, which is consistent with the predictions from the SHF approach using different Skyrme interactions, as shown in Table II, where one can see that only 5 Skyrme interactions (i.e., RATP, SII, SIV, SKa, and SV) among the 63 Skyrme interactions have $E_{\text {sym }, 4}\left(\rho_{0}\right)$ larger than $1 \mathrm{MeV}$ (but still less than $2 \mathrm{MeV}$ ). The value of $E_{\text {sym }, 4}\left(\rho_{0}\right)=0.62 \mathrm{MeV}$ is further consistent with the value of $0.57 \mathrm{MeV}$ predicted by the MSL model using Eq. (70). These results thus confirm the empirical parabolic law that the

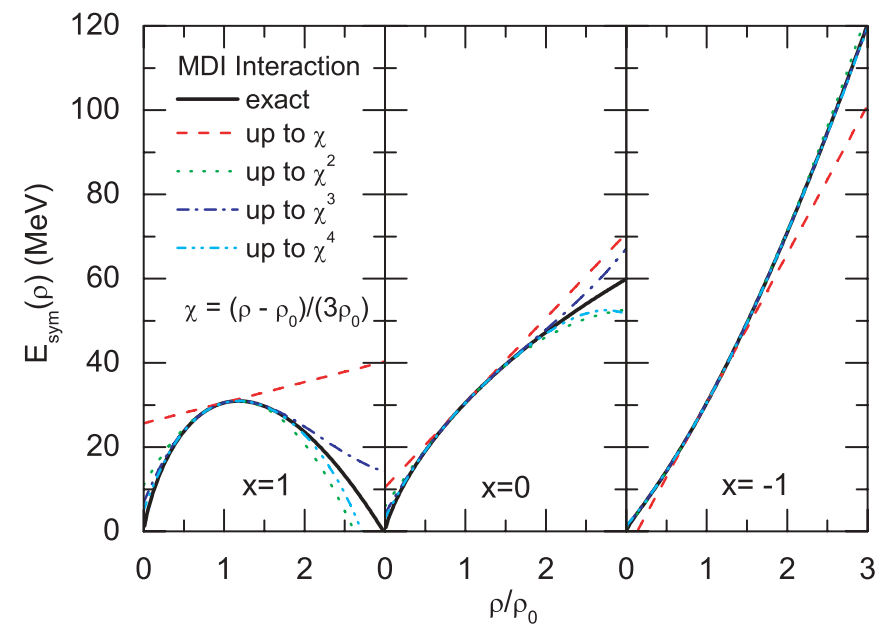

FIG. 2. (Color online) Density dependence of symmetry energy using the MDI interaction with $x=1$ (left), 0 (middle), and -1 (right) together with corresponding results obtained by using Eq. (11) up to $\chi, \chi^{2}, \chi^{3}$, and $\chi^{4}$, respectively. 
TABLE II. The characteristic parameters $E_{\text {sym }}\left(\rho_{0}\right)(\mathrm{MeV}), L(\mathrm{MeV}), K_{\mathrm{sym}}(\mathrm{MeV}), J_{\mathrm{sym}}(\mathrm{MeV}), E_{\mathrm{sym}, 4}\left(\rho_{0}\right)(\mathrm{MeV})$, $L_{\mathrm{sym}, 4}(\mathrm{MeV}), K_{\mathrm{sym}, 4}(\mathrm{MeV}), m_{s, 0}^{*} / m$, and $m_{v, 0}^{*} / m$ at saturation density for the MDI interaction with $x=-1,0$, and 1 and the SHF predictions with 63 standard Skyrme interactions.

\begin{tabular}{|c|c|c|c|c|c|c|c|c|c|}
\hline Force & $E_{\text {sym }}\left(\rho_{0}\right)$ & $L$ & $K_{\mathrm{sym}}$ & $J_{\mathrm{sym}}$ & $E_{\text {sym }, 4}\left(\rho_{0}\right)$ & $L_{\text {sym }, 4}$ & $K_{\mathrm{sym}, 4}$ & $m_{s, 0}^{*} / m$ & $m_{v, 0}^{*} / m$ \\
\hline MDI(1) & 30.5 & 14.7 & -264.0 & 660.0 & 0.62 & 0.53 & -4.82 & 0.67 & 0.54 \\
\hline $\operatorname{MDI}(0)$ & 30.5 & 60.2 & -81.7 & 295.3 & 0.62 & 0.53 & -4.82 & 0.67 & 0.54 \\
\hline $\operatorname{MDI}(-1)$ & 30.5 & 105.8 & 100.7 & -69.3 & 0.62 & 0.53 & -4.82 & 0.67 & 0.54 \\
\hline$Z$ & 26.8 & -49.7 & -657.9 & 495.2 & 0.78 & 2.56 & 1.47 & 0.84 & 0.73 \\
\hline$E_{\sigma}$ & 26.4 & -36.9 & -457.8 & 880.0 & 0.88 & 3.04 & 2.38 & 0.84 & 0.70 \\
\hline$E$ & 27.7 & -31.3 & -570.7 & 448.6 & 0.80 & 2.66 & 1.67 & 0.87 & 0.74 \\
\hline$Z_{\sigma}$ & 26.7 & -29.4 & -401.4 & 883.1 & 0.91 & 3.14 & 2.58 & 0.78 & 0.66 \\
\hline SVI & 26.9 & -7.3 & -471.3 & 146.0 & 0.67 & 2.08 & 0.76 & 0.95 & 0.81 \\
\hline$Z_{\sigma}^{*}$ & 28.8 & -4.5 & -332.6 & 725.1 & 0.92 & 3.24 & 2.79 & 0.77 & 0.65 \\
\hline $\mathrm{SkSC} 4$ & 28.8 & -2.2 & -329.5 & 708.3 & 0.46 & 0.91 & -1.85 & 1.00 & 1.00 \\
\hline SI & 29.2 & 1.2 & -461.8 & 141.4 & 0.70 & 2.16 & 0.73 & 0.91 & 0.80 \\
\hline BSk3 & 27.9 & 6.8 & -306.9 & 550.3 & 0.71 & 2.19 & 0.76 & 1.12 & 0.89 \\
\hline BSk1 & 27.8 & 7.2 & -281.8 & 606.4 & 0.43 & 0.79 & -2.04 & 1.05 & 1.05 \\
\hline BSk2 & 28.0 & 8.0 & -297.0 & 557.9 & 0.71 & 2.18 & 0.74 & 1.04 & 0.86 \\
\hline MSk7 & 27.9 & 9.4 & -274.6 & 592.1 & 0.43 & 0.79 & -2.04 & 1.05 & 1.05 \\
\hline SIII & 28.1 & 9.9 & -393.7 & 130.4 & 0.83 & 2.89 & 2.34 & 0.76 & 0.66 \\
\hline BSk4 & 28.0 & 12.5 & -265.9 & 558.4 & 0.61 & 1.70 & -0.22 & 0.92 & 0.85 \\
\hline BSk8 & 28.0 & 14.9 & -220.9 & 624.9 & 0.43 & 0.78 & -2.09 & 0.80 & 0.87 \\
\hline BSk6 & 28.0 & 16.8 & -215.2 & 603.5 & 0.45 & 0.89 & -1.85 & 0.80 & 0.86 \\
\hline BSk7 & 28.0 & 18.0 & -209.4 & 598.2 & 0.42 & 0.77 & -2.08 & 0.80 & 0.87 \\
\hline SKP & 30.0 & 19.6 & -266.8 & 508.6 & 0.94 & 3.33 & 2.96 & 1.00 & 0.74 \\
\hline BSk5 & 28.7 & 21.4 & -240.3 & 499.9 & 0.64 & 1.83 & 0.04 & 0.92 & 0.84 \\
\hline SKXm & 31.2 & 32.1 & -242.8 & 428.7 & 0.88 & 3.02 & 2.40 & 0.97 & 0.75 \\
\hline RATP & 29.2 & 32.4 & -191.2 & 440.6 & 1.06 & 3.94 & 4.21 & 0.67 & 0.56 \\
\hline SKX & 31.1 & 33.2 & -252.1 & 379.7 & 0.89 & 3.10 & 2.61 & 0.99 & 0.75 \\
\hline SKXce & 30.1 & 33.5 & -238.4 & 356.9 & 0.89 & 3.12 & 2.65 & 1.01 & 0.75 \\
\hline BSk15 & 30.0 & 33.6 & -194.4 & 466.5 & 0.64 & 1.84 & 0.03 & 0.80 & 0.77 \\
\hline BSk16 & 30.0 & 34.9 & -187.4 & 461.9 & 0.60 & 1.66 & -0.31 & 0.80 & 0.78 \\
\hline BSk10 & 30.0 & 37.2 & -194.9 & 397.0 & 0.69 & 2.08 & 0.50 & 0.92 & 0.81 \\
\hline SGII & 26.8 & 37.6 & -145.9 & 330.4 & 0.87 & 3.01 & 2.39 & 0.79 & 0.67 \\
\hline BSk12 & 30.0 & 38.0 & -191.4 & 392.5 & 0.68 & 2.02 & 0.40 & 0.92 & 0.82 \\
\hline BSk11 & 30.0 & 38.4 & -189.8 & 390.1 & 0.67 & 2.01 & 0.37 & 0.92 & 0.82 \\
\hline SLy10 & 32.0 & 38.7 & -142.2 & 591.2 & 0.37 & 0.50 & -2.60 & 0.68 & 0.80 \\
\hline BSk13 & 30.0 & 38.8 & -187.9 & 386.6 & 0.67 & 2.00 & 0.37 & 0.92 & 0.82 \\
\hline BSk9 & 30.0 & 39.9 & -145.3 & 475.8 & 0.37 & 0.47 & -2.71 & 0.80 & 0.91 \\
\hline BSk14 & 30.0 & 43.9 & -152.0 & 388.3 & 0.60 & 1.65 & -0.33 & 0.80 & 0.78 \\
\hline SLy230a & 32.0 & 44.3 & -98.2 & 602.9 & 0.06 & -1.07 & -5.80 & 0.70 & 1.00 \\
\hline SLy6 & 31.2 & 45.2 & -112.5 & 511.3 & 0.38 & 0.55 & -2.54 & 0.69 & 0.80 \\
\hline SLy8 & 31.4 & 45.3 & -116.5 & 511.4 & 0.40 & 0.64 & -2.39 & 0.70 & 0.80 \\
\hline SLy4 & 31.8 & 45.4 & -119.9 & 521.0 & 0.40 & 0.63 & -2.40 & 0.69 & 0.80 \\
\hline SLy0 & 31.5 & 45.4 & -116.8 & 510.6 & 0.40 & 0.64 & -2.39 & 0.70 & 0.80 \\
\hline SLy3 & 32.1 & 45.5 & -122.1 & 526.2 & 0.40 & 0.63 & -2.41 & 0.70 & 0.80 \\
\hline SKM $^{*}$ & 30.0 & 45.8 & -155.9 & 330.5 & 0.94 & 3.32 & 2.97 & 0.79 & 0.65 \\
\hline SLy230b & 32.0 & 46.0 & -119.7 & 521.5 & 0.40 & 0.61 & -2.43 & 0.69 & 0.80 \\
\hline SLy7 & 32.4 & 48.1 & -114.3 & 516.6 & 0.38 & 0.55 & -2.53 & 0.69 & 0.80 \\
\hline SLy2 & 32.3 & 48.8 & -113.5 & 502.9 & 0.40 & 0.63 & -2.39 & 0.70 & 0.80 \\
\hline SLy1 & 32.5 & 48.8 & -115.7 & 508.5 & 0.40 & 0.63 & -2.40 & 0.70 & 0.80 \\
\hline SKM & 30.7 & 49.3 & -148.8 & 323.3 & 0.91 & 3.19 & 2.71 & 0.79 & 0.66 \\
\hline SII & 34.2 & 50.0 & -265.7 & 104.7 & 1.10 & 4.21 & 4.94 & 0.58 & 0.50 \\
\hline SLy5 & 32.7 & 50.3 & -111.9 & 499.2 & 0.40 & 0.63 & -2.40 & 0.70 & 0.80 \\
\hline SLy9 & 32.1 & 55.4 & -81.3 & 461.8 & 0.33 & 0.32 & -2.88 & 0.67 & 0.80 \\
\hline SkI6 & 29.9 & 59.2 & -46.8 & 378.1 & 0.28 & 0.04 & -3.55 & 0.64 & 0.80 \\
\hline SkI4 & 29.5 & 60.4 & -40.6 & 351.2 & 0.30 & 0.15 & -3.36 & 0.65 & 0.80 \\
\hline SIV & 31.2 & 63.5 & -136.7 & 79.5 & 1.37 & 5.51 & 7.50 & 0.47 & 0.41 \\
\hline SGI & 28.3 & 63.9 & -52.0 & 194.5 & 0.86 & 3.00 & 2.36 & 0.61 & 0.57 \\
\hline
\end{tabular}


TABLE II. (Continued.)

\begin{tabular}{lcccccrrrr}
\hline \hline Force & $E_{\text {sym }}\left(\rho_{0}\right)$ & \multicolumn{1}{c}{$L$} & \multicolumn{1}{c}{$K_{\text {sym }}$} & \multicolumn{1}{c}{$J_{\text {sym }}$} & $E_{\text {sym }, 4}\left(\rho_{0}\right)$ & $L_{\text {sym }, 4}$ & $K_{\text {sym }, 4}$ & $m_{s, 0}^{*} / m$ & $m_{v, 0}^{*} / m$ \\
\hline SKO $^{*}$ & 32.1 & 69.7 & -77.5 & 221.4 & 0.55 & 1.38 & -0.90 & 0.90 & 0.87 \\
SkMP & 29.7 & 69.8 & -50.3 & 159.7 & 0.93 & 3.31 & 3.00 & 0.65 & 0.58 \\
SKa & 32.9 & 74.6 & -78.5 & 174.5 & 1.13 & 4.33 & 5.06 & 0.61 & 0.51 \\
SKO & 32.0 & 79.6 & -42.3 & 130.0 & 0.59 & 1.57 & -0.53 & 0.89 & 0.85 \\
$R_{\sigma}$ & 30.6 & 85.7 & -9.1 & 22.2 & 0.85 & 2.88 & 2.13 & 0.78 & 0.68 \\
$G_{\sigma}$ & 31.4 & 94.0 & 14.0 & -26.7 & 0.85 & 2.87 & 2.12 & 0.78 & 0.68 \\
SKT4 & 35.5 & 94.1 & -24.5 & 97.8 & 0.45 & 0.91 & -1.83 & 1.00 & 1.00 \\
SV & 32.8 & 96.1 & 24.2 & 48.0 & 1.70 & 7.18 & 10.77 & 0.38 & 0.33 \\
SkI3 & 34.8 & 100.5 & 73.0 & 211.5 & 0.12 & -0.74 & -5.10 & 0.58 & 0.82 \\
SkI2 & 33.4 & 104.3 & 70.7 & 51.6 & 0.37 & 0.48 & -2.66 & 0.68 & 0.80 \\
SkI5 & 36.6 & 129.3 & 159.6 & 11.7 & 0.12 & -0.72 & -5.04 & 0.58 & 0.80 \\
\hline \hline
\end{tabular}

higher order (including fourth-order) contributions of $\delta$ in the EOS of asymmetric nuclear matter are usually very small and negligible, as mentioned previously. Furthermore, similarly to the cases shown in Figs. 1 and 2, including terms up to $\chi^{2}$ in Eq. (16) can approximate very well the exact fourth-order symmetry energy for the density region between about $0.5 \rho_{0}$ and $1.5 \rho_{0}$ and including higher order terms of $\chi^{3}$ and $\chi^{4}$ improves significantly the results at low densities and up to about $2 \rho_{0}$.

These results thus indicate that generally one needs higher order terms in $\chi$ (higher than fourth order) to describe the EOS of asymmetric nuclear matter in the high-density region (above $2 \rho_{0}$ ). We note that the conclusions obtained with the MDI interaction are also valid for the SHF approach and the MSL model. These features imply that it is very difficult to obtain correct information on the high-density behavior of the EOS for asymmetric nuclear matter based on the characteristic parameters obtained at normal nuclear density $\rho_{0}$. At this point, it should be mentioned that the transport model analysis of heavy-ion collisions at intermediate and high energies as well as the astrophysical observations, especially on the properties

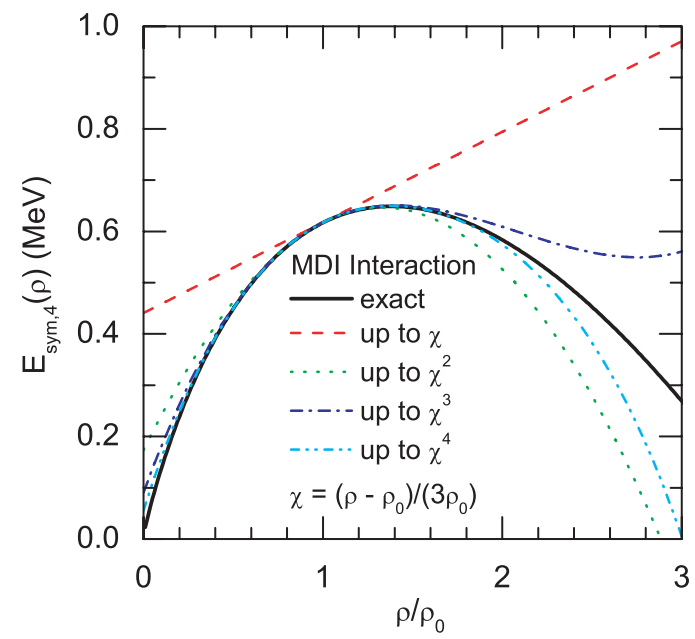

FIG. 3. (Color online) Density dependence of the fourth-order symmetry energy $E_{\text {sym }, 4}(\rho)$ using the MDI interaction together with results obtained by using Eq. (16) up to $\chi, \chi^{2}, \chi^{3}$, and $\chi^{4}$, respectively. of compact stars, provide unique tools to extract information on the EOS of asymmetric nuclear matter at high densities $[8,12,15,26,114]$.

\section{B. Isospin dependence of the saturation properties of asymmetric nuclear matter}

In the following, we show the results on the saturation properties of asymmetric nuclear matter (i.e., the saturation density as well as the binding energy and incompressibility at saturation density). Especially, we investigate their isospin dependence and study whether the higher order terms in the isospin asymmetry $\delta\left(\delta^{4}\right.$ term) are important for the description of the saturation properties of asymmetric nuclear matter.

\section{The saturation density}

The saturation density is a basic quantity of asymmetric nuclear matter. To see the symmetry energy dependence of the saturation density of asymmetric nuclear matter, we use here the MDI interaction with $x=1,0$, and -1 . The density dependence of the symmetry energy from this interaction is shown in Fig. 4. Also included in Fig. 4 are the results from the widely used APR (Akmal-Pandharipande-Ravenhall) prediction [115] and the phenomenological MSL model prediction with $y=-15,-7.5$, and 0.75 (the results of which will be discussed later). It is seen that the APR prediction for the symmetry energy resembles very well that from the MDI interaction with $x=0$ up to about $3.5 \rho_{0}$.

Using the MDI interaction with $x=1,0$, and -1 , we have calculated the density and isospin asymmetry dependence of the binding energy per nucleon of asymmetric nuclear matter, and the results are shown in Fig. 5. Further indicated in Fig. 5 are corresponding saturation points in the $E-\rho$ plane. One can see that different symmetry energies lead to different equations of state of pure neutron matter $(\delta=1)$ as expected. In particular, the EOS of pure neutron matter for $x=-1$ is bounded at low densities. In addition, different symmetry energies lead to rather different behaviors for the saturation points in the $E$ - $\rho$ plane.

To see more clearly the isospin dependence of the saturation density, we show in Fig. 6 the saturation density $\rho_{\text {sat }}(\delta)$ as a function of $\delta^{2}$ in the MDI interaction with $x=1,0$, and -1 , 


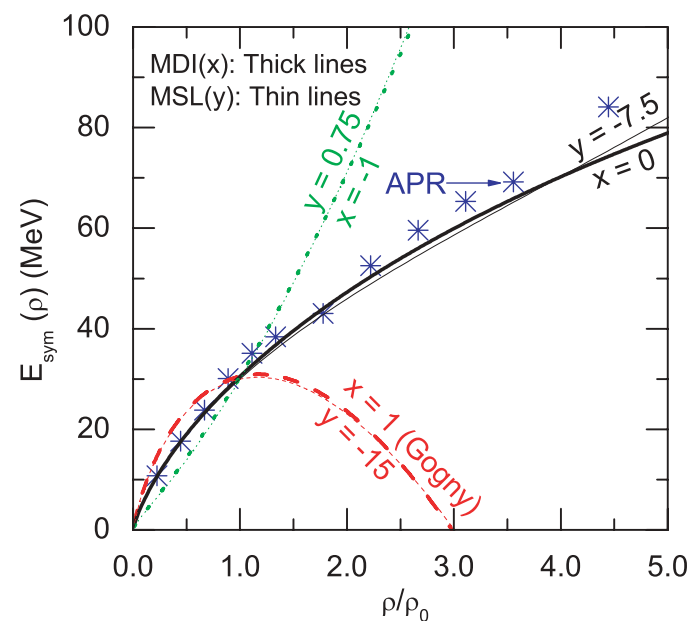

FIG. 4. (Color online) Density dependence of the symmetry energy from the MDI interaction with $x=1,0$, and -1 . The results from the widely used APR prediction [115] and the phenomenological MSL model prediction with $y=-15,-7.5$, and 0.75 are also included for comparison.

respectively. The exact saturation density $\rho_{\text {sat }}(\delta)$ is obtained from Eqs. (21) and (43). Corresponding results from Eq. (23) including terms up to $\delta^{2}$ and up to $\delta^{4}$, respectively, are also included for comparison. The results indicate that the saturation density generally decreases with isospin asymmetry and more neutron rich nuclear matter has lower saturation density. In addition, for the stiffer symmetry energy $(x=-1)$, the nuclear matter can be bounded even for pure neutron matter (the corresponding saturation density being about $0.3 \rho_{0}$ ). The inset in Fig. 6 displays corresponding results at smaller isospin asymmetries with $\delta^{2} \leqslant 0.1$, which is relevant to the properties of finite nuclei. In the small isospin asymmetry region $\left(\delta^{2} \leqslant 0.1\right)$, the saturation density $\rho_{\text {sat }}(\delta)$ displays a linear dependence on $\delta^{2}$ and Eq. (23) including terms up to $\delta^{2}$ thus approximates very well the exact $\rho_{\text {sat }}(\delta)$. Furthermore, how

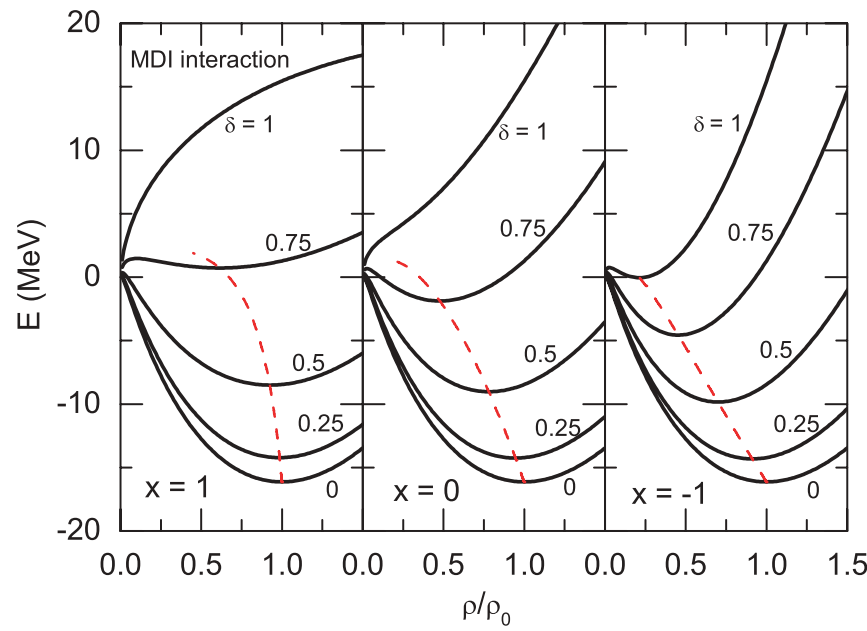

FIG. 5. (Color online) The density and isospin asymmetry dependence of the binding energy per nucleon for asymmetric nuclear matter in the MDI interaction with $x=1,0$, and -1 . The saturation points at different isospin asymmetries are also indicated.

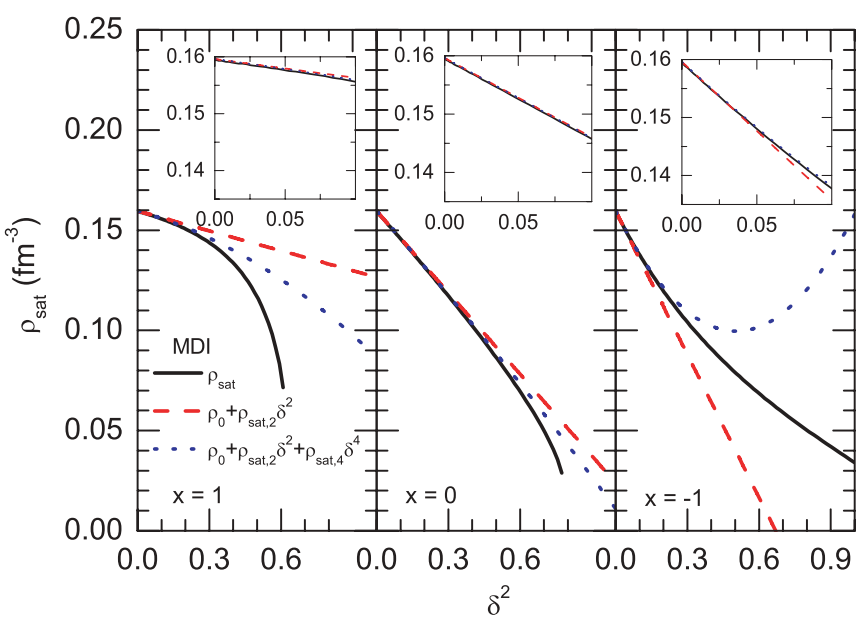

FIG. 6. (Color online) The saturation density $\rho_{\text {sat }}$ as a function of $\delta^{2}$ in the MDI interaction with $x=1,0$, and -1 . Corresponding results from Eq. (23) including terms up to $\delta^{2}$ and up to $\delta^{4}$, respectively, are also included for comparison. The insets display corresponding results at smaller isospin asymmetries with $\delta^{2} \leqslant 0.1$.

$\rho_{\text {sat }}(\delta)$ decreases with increasing $\delta$ depends on the stiffness of symmetry energy, with the softer symmetry energy having a weaker dependence, and this feature can be easily understood from Eq. (24), which indicates that the slope of $\rho_{\text {sat }}(\delta)$ with respect to $\delta^{2}$ is proportional to $-\frac{3 L}{K_{0}} \rho_{0}$. In addition, at larger isospin asymmetries with $\delta^{2} \geqslant 0.3$, including higher order $\delta$ terms up to $\delta^{4}$ in Eq. (23) still deviates significantly from the exact $\rho_{\text {sat }}(\delta)$ and higher order terms of $\delta$ are thus necessary [except for the case of $x=0$ where Eq. (23) with terms up to $\delta^{4}$ gives a good approximation to the exact $\rho_{\text {sat }}(\delta)$ in the whole $\delta$ region where the asymmetric matter can still be bounded]. These features imply that higher order terms in $\delta$ may be important for the determination of the saturation density of nuclear matter in a very neutron rich nuclear environment, such as inside a neutron star.

\section{Binding energy at saturation density}

The isospin dependence of the binding energy per nucleon of asymmetric nuclear matter at saturation density $E_{\mathrm{sat}}(\delta)$ is shown in Fig. 7 as a function of $\delta^{2}$ for the MDI interaction with $x=1,0$, and -1 . The exact $E_{\text {sat }}(\delta)$ is obtained from Eqs. (21) and (43). Corresponding results from Eq. (27) including terms up to $\delta^{2}$ and up to $\delta^{4}$, respectively, are also included for comparison. It is seen that $E_{\text {sat }}(\delta)$ generally increases with increasing isospin asymmetry. The results at smaller isospin asymmetries with $\delta^{2} \leqslant 0.1$ are shown in the inset of Fig. 7 and it is seen that $E_{\text {sat }}(\delta)$ displays a linear dependence on $\delta^{2}$ and therefore can be very well approximated by Eq. (27) including terms up to $\delta^{2}$. We note that the rate at which $E_{\text {sat }}(\delta)$ increases with $\delta^{2}$ at small $\delta^{2}$ is determined uniquely by $E_{\mathrm{sym}}\left(\rho_{0}\right)$, as shown in Eq. (27). Also, it is seen that including higher order $\delta$ terms up to $\delta^{4}$ in Eq. (27) give a good approximation to the exact $E_{\text {sat }}(\delta)$ in the whole $\delta$ region [except for the case of $x=-1$ where Eq. (27) including terms up to $\delta^{4}$ still deviates significantly from the exact $E_{\text {sat }}(\delta)$ at larger isospin asymmetries with $\delta^{2} \geqslant 0.3$ and higher order terms of $\delta$ are thus needed]. 


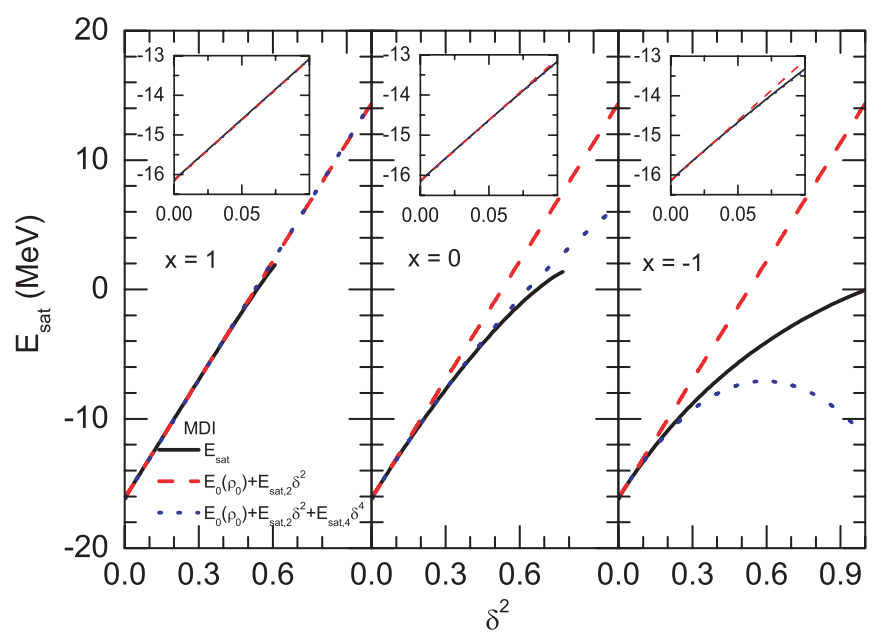

FIG. 7. (Color online) Same as Fig. 6 but for the binding energy at saturation density, $E_{\mathrm{sat}}$.

\section{Incompressibility at saturation density}

Shown in Fig. 8 is the incompressibility at saturation density $K_{\text {sat }}(\delta)$ as a function of $\delta^{2}$ for the MDI interaction with $x=1,0$, and -1 . The exact $K_{\text {sat }}(\delta)$ is obtained from Eqs. (21), (32), and (43), and corresponding results from Eq. (34) including terms up to $\delta^{2}$ and up to $\delta^{4}$ are also included for comparison. It is seen that $K_{\text {sat }}(\delta)$ generally decreases with increasing isospin asymmetry and more neutron rich nuclear matter has smaller incompressibility. This feature is consistent with earlier calculations based on microscopic many-body approaches [116]. The softening of the incompressibility of asymmetric nuclear matter with increasing isospin asymmetry may have important implications in understanding the mechanism for supernovae explosions [116,117]. Corresponding results at smaller isospin asymmetries with $\delta^{2} \leqslant 0.1$ are given in the inset of Fig. 8, which shows that Eq. (34) including terms up to $\delta^{2}$ approximates very well the exact $K_{\text {sat }}(\delta)$ as $K_{\text {sat }}(\delta)$ displays a good linear correlation with $\delta^{2}$. The decreasing rate

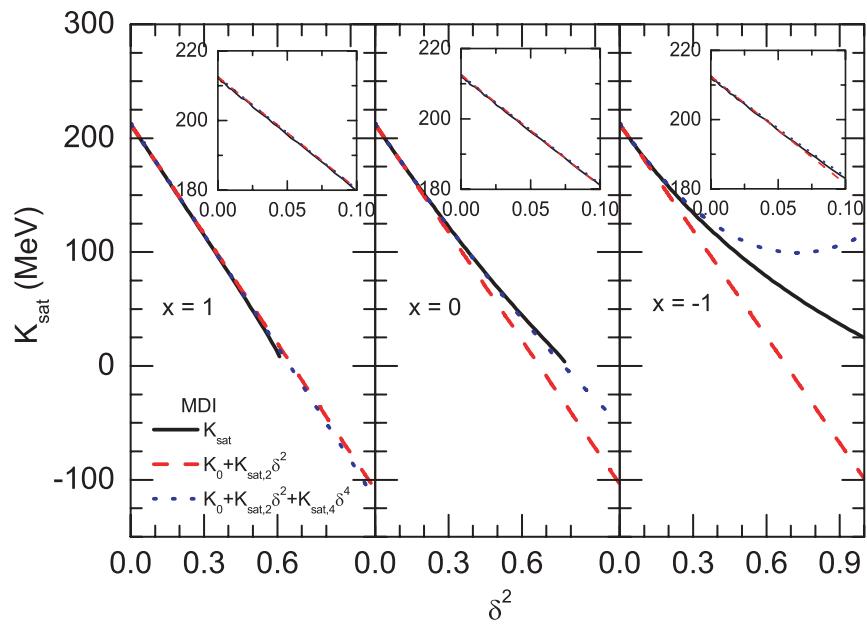

FIG. 8. (Color online) Same as Fig. 6 but for the incompressibility at saturation density, $K_{\text {sat }}$. of $K_{\text {sat }}(\delta)$ with increasing $\delta^{2}$ at small $\delta^{2}$ is determined by the parameter $K_{\text {sat, } 2}$, as shown in Eq. (34), which depends on the characteristic parameters $J_{0}, K_{0}, L$, and $K_{\text {sym }}$. In addition, including higher order $\delta$ terms up to $\delta^{4}$ in Eq. (34) is seen to give a good approximation to the exact $K_{\text {sat }}(\delta)$ in the whole $\delta$ region [except for the case of $x=-1$ where Eq. (34) including terms up to $\delta^{4}$ deviates significantly from the exact $K_{\text {sat }}(\delta)$ at larger isospin asymmetries with $\delta^{2} \geqslant 0.3$ and higher order terms of $\delta$ are thus important].

These results indicate that the saturation properties of asymmetric nuclear matter (i.e., the saturation density as well as the binding energy and incompressibility at saturation density) exhibit a good linear correlation with $\delta^{2}$ at smaller isospin asymmetries with $\delta^{2} \leqslant 0.1$, which is relevant to the properties of finite heavy nuclei. However, depending on the stiffness of nuclear symmetry energy, higher order terms in $\delta$ ( $\delta^{4}$ and higher order terms) may become important for describing reasonably the saturation properties of asymmetric nuclear matter at larger isospin asymmetries with $\delta^{2} \geqslant 0.3$. The importance of higher order isospin asymmetry terms for the stiffer symmetry energy has also been observed in previous studies on the transition density in neutron stars [72,73]. In addition, the saturation density and the incompressibility at saturation density generally decrease with the magnitude of isospin asymmetry whereas the binding energy at saturation density shows an opposite behavior. Again, we note that the conclusions obtained here from the MDI interaction are also valid for the SHF approach and the MSL model. Our results are further consistent with the very recent study based on the RMF model [118].

\section{Constraining the $K_{\text {sat,2 }}$ parameter from the phenomenological MSL model}

\section{General information on the $K_{\mathrm{sat}, 2}$ parameter}

As just shown, the $K_{\mathrm{sat}, 2}$ parameter essentially characterizes the isospin dependence of the incompressibility of asymmetric nuclear matter at saturation density and the higher order parameters (e.g., $K_{\text {sat }, 4}$ ) are only important for extremely neutron rich (or proton rich) nuclear matter with stiffer nuclear symmetry energies. Actually, it can be seen from Table I that the magnitude (absolute values) of $K_{\mathrm{sat}, 2}$ is generally much larger than that of $K_{\mathrm{sat}, 4}$ for the MDI interaction with $x=1$, 0 , and -1 and the 63 standard Skyrme interactions considered in the present work. Shown in Fig. 9 are the absolute values of $K_{\text {sat, } 2}$ and $K_{\text {asy }}$ as well as the value of $K_{\text {sat }, 4}$ as functions of $L$ for the MDI interaction with $x=1,0,-1$ and the 63 standard Skyrme interactions considered in the present work. It is seen that these values of $K_{\mathrm{sat}, 2}$ can be nicely expressed as $-400 \pm 120 \mathrm{MeV}$. The magnitude of the $K_{\text {asy }}$ parameter is generally larger than that of the $K_{\text {sat, } 2}$ parameter, especially for the stiffer symmetry energies (larger $L$ values), which indicates that the higher order $J_{0}$ parameter is important, as will be discussed later. Furthermore, the absolute values of $K_{\text {sat, } 2}$ are clearly much larger than that of $K_{\mathrm{sat}, 4}$ except that at very large $L$ values the absolute values of $K_{\text {sat }, 4}$ may become larger and comparable with that of $K_{\mathrm{sat}, 2}$. This feature is consistent with 


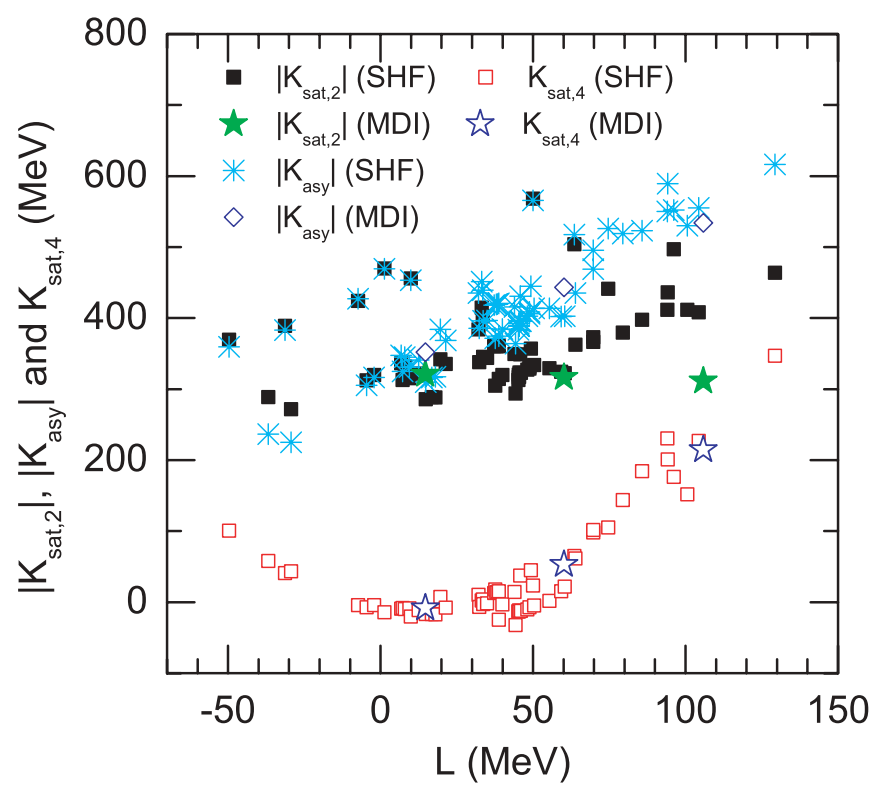

FIG. 9. (Color online) The absolute values of $K_{\text {sat, } 2}$ and $K_{\text {asy }}$ and the value of $K_{\mathrm{sat}, 4}$ as functions of $L$ for the MDI interaction with $x=1,0$, and -1 and the 63 standard Skyrme interactions considered in the present work.

the results shown in Fig. 8, where the higher order terms are seen to be only important for the stiffer symmetry energies.

It is generally believed that information on $K_{\text {sat, } 2}$ can be extracted experimentally by measuring the GMR in neutronrich nuclei [119]. Usually, one can define a finite nucleus incompressibility $K_{A}(N, Z)$ for a nucleus with $N$ neutrons and $Z$ protons $(A=N+Z)$ by the energy of GMR $E_{\mathrm{GMR}}$, that is,

$$
E_{\mathrm{GMR}}=\sqrt{\frac{\hbar^{2} K_{A}(N, Z)}{m\left\langle r^{2}\right\rangle}},
$$

where $m$ is the nucleon mass and $\left\langle r^{2}\right\rangle$ is the mean square mass radius of the nucleus in the ground state. Similarly to the semiempirical mass formula, the finite nucleus incompressibility $K_{A}(N, Z)$ can be expanded as [119]

$$
\begin{aligned}
K_{A}(N, Z)= & K_{0}+K_{\text {surf }} A^{-1 / 3}+K_{\text {curv }} A^{-2 / 3} \\
& +\left(K_{\tau}+K_{\text {ss }} A^{-1 / 3}\right)\left(\frac{N-Z}{A}\right)^{2} \\
& +K_{\text {Coul }} \frac{Z^{2}}{A^{4 / 3}}+\cdots
\end{aligned}
$$

Neglecting the $K_{\text {curv }}$ term, the $K_{\mathrm{ss}}$ term, and other higher order terms in Eq. (72), one can express the finite nucleus incompressibility $K_{A}(N, Z)$ as

$$
\begin{aligned}
K_{A}(N, Z)= & K_{0}+K_{\text {surf }} A^{-1 / 3}+K_{\tau}\left(\frac{N-Z}{A}\right)^{2} \\
& +K_{\text {Coul }} \frac{Z^{2}}{A^{4 / 3}},
\end{aligned}
$$

where $K_{0}, K_{\text {surf }}, K_{\tau}$, and $K_{\text {Coul }}$ represent the volume, surface, symmetry, and Coulomb terms, respectively. The $K_{\tau}$ parameter is usually thought to be equivalent to the $K_{\mathrm{sat}, 2}$ parameter.
However, we would like to stress here that the $K_{\text {sat, } 2}$ parameter is a theoretically well-defined physical property of asymmetric nuclear matter as shown previously whereas the value of the $K_{\tau}$ parameter may depend on the details of the truncation scheme in Eq. (72). As shown in Ref. [120], $K_{\tau}$ may be related to the isospin-dependent part of the surface properties of finite nuclei, especially the surface symmetry energy. Therefore, caution is needed to interpret the $K_{\tau}$ parameter as the $K_{\text {sat, } 2}$ parameter (a point we will return to later).

Earlier attempts based on this method have given widely different values for the $K_{\tau}$ parameter. For example, a value of $K_{\tau}=-320 \pm 180 \mathrm{MeV}$ with a large uncertainty was obtained in Ref. [121] from a systematic study of the GMR in the isotopic chains of $\mathrm{Sn}$ and $\mathrm{Sm}$. In this analysis, the value of $K_{0}$ was found to be $300 \pm 25 \mathrm{MeV}$, which is somewhat larger than the commonly accepted value of $240 \pm 20 \mathrm{MeV}$. In a later study, an even less stringent constraint of $-566 \pm 1350<$ $K_{\tau}<139 \pm 1617 \mathrm{MeV}$ was extracted from the GMR of finite nuclei, depending on the mass region of nuclei and the number of parameters used in parametrizing the incompressibility of finite nuclei [122]. More recently, a much more stringent constraint of $K_{\tau}=-550 \pm 100 \mathrm{MeV}$ has been obtained in Refs. [22,23] from measurements of the isotopic dependence of the GMR in even-A Sn isotopes.

\section{Correlation between $J_{0}$ and $K_{0}$}

As shown in Eq. (35), the $K_{\text {sat, } 2 \text { parameter is completely }}$ determined by the four characteristic parameters $K_{0}, J_{0}, L$, and $K_{\text {sym }}$ at normal nuclear density. It thus would be interesting to estimate the possible value of $K_{\text {sat, } 2}$ from knowledge of $K_{0}, J_{0}, L$, and $K_{\text {sym }}$. For the incompressibility of symmetric nuclear matter at its saturation density $\rho_{0}$, the transport model analyses on experimental data from subthreshold kaon production in heavy-ion collisions favor a soft equation of state [26,123,124]. More recently, the value of $K_{0}$ has been more stringently determined to be $240 \pm 20 \mathrm{MeV}$ from the nuclear GMR data [16,21-24].

Although the $K_{0}$ parameter has been relatively well determined, the $J_{0}$ parameter is poorly known, and there is actually no experimental information on the $J_{0}$ parameter. In the MSL model, one can easily calculate from Eq. (57) the $J_{0}$ parameter as

$$
\begin{aligned}
J_{0}= & \left.27 \rho_{0}^{3} \frac{d^{3} E_{0}(\rho)}{d^{3} \rho}\right|_{\rho=\rho_{0}} \\
= & \frac{1}{E_{\text {kin }}^{0}-3 E_{0}-2 C}\left[K_{0}^{2}+\left(18 E_{0}-2 E_{\text {kin }}^{0}-8 C\right) K_{0}\right. \\
& \left.+12 E_{\text {kin }}^{0} E_{0}+6 C\left(3 E_{\text {kin }}^{0}-25 E_{0}\right)\right] .
\end{aligned}
$$

In particular, for $m_{s, 0}^{*}=0.8 m$, we have

$$
J_{0}=\frac{1}{59.1}\left(K_{0}^{2}-376.4 K_{0}+11214.6\right)(\mathrm{MeV}),
$$

whereas we have

$$
J_{0}=\frac{1}{70.1}\left(K_{0}^{2}-332.2 K_{0}-4243.2\right)(\mathrm{MeV})
$$




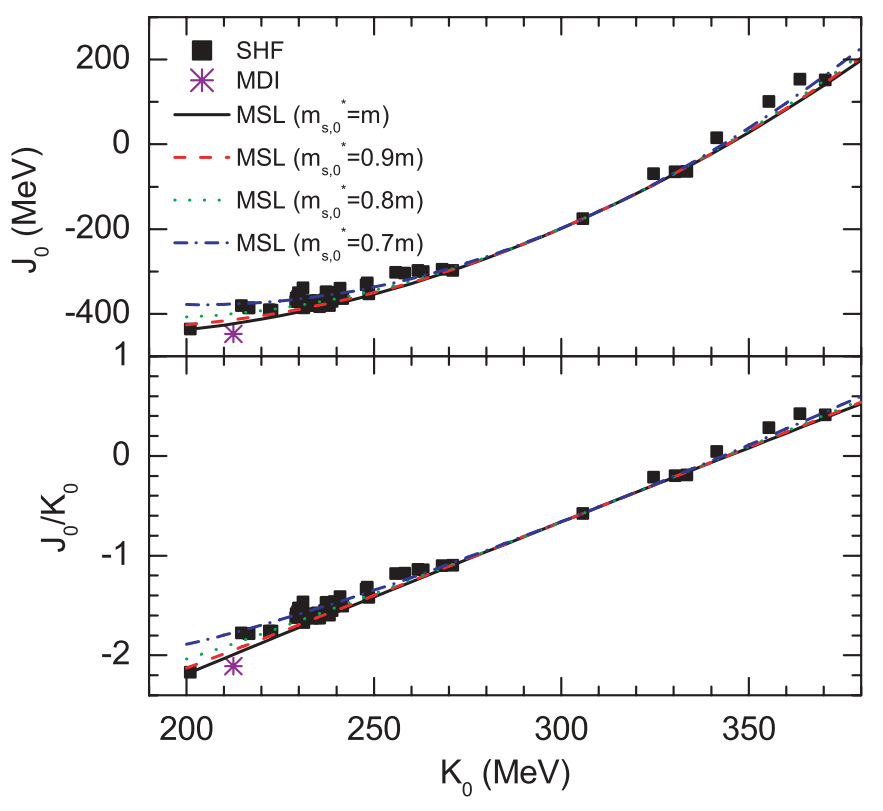

FIG. 10. (Color online) The third derivative parameter $J_{0}$ and its ratio $J_{0} / K_{0}$ to the incompressibility $K_{0}$ of symmetric nuclear matter at saturation density as functions of $K_{0}$ from the MSL model, the MDI interaction, and the SHF prediction with the 63 Skyrme interactions shown in Table I.

for $m_{s, 0}^{*}=m$ (and then $\left.C=0\right)$, and

$$
J_{0}=\frac{1}{51.2}\left(K_{0}^{2}-408.0 K_{0}+22252.0\right)(\mathrm{MeV})
$$

for $m_{s, 0}^{*}=0.7 m$ and $C=9.47 \mathrm{MeV}$. In these equations, the units of $K_{0}$ are $\mathrm{MeV}$. Therefore, the $J_{0}$ parameter in the phenomenological MSL model is a quadratic function of the $K_{0}$ parameter.

Shown in Fig. 10 are $J_{0}$ and $J_{0} / K_{0}$ as functions of $K_{0}$ in the MSL model for different values of $m_{s, 0}^{*}=m, 0.9 m, 0.8 m$, and $0.7 \mathrm{~m}$. Also included in Fig. 10 are corresponding results from the MDI interaction and the SHF prediction with the 63 Skyrme interactions shown in Table I. It is seen that the correlation between $J_{0}$ and $K_{0}$ is similar among these three different models. Also, all three models show an approximately linear correlation between $J_{0} / K_{0}$ and $K_{0}$. This linear correlation can be easily understood from Eqs. (75), (76), and (77). For example, on the r.h.s of Eq. (75), the last term is small compared with the first two terms, and thus one has $J_{0} \approx \frac{1}{59.1}\left(K_{0}^{2}-376.4 K_{0}\right)$, and then $J_{0} / K_{0} \approx \frac{1}{59.1}\left(K_{0}-376.4\right)$ with the units of $K_{0}$ in $\mathrm{MeV}$. The linear dependence of $J_{0} / K_{0}$ on $K_{0}$ becomes better for larger values of $m_{s, 0}^{*}$, which usually leads to smaller values for the last two terms on the r.h.s of Eq. (74), as shown in Eq. (76). We note that the correlation between $J_{0}$ and $K_{0}$ obtained in the present work is also consistent with the early finding by Pearson [125]. In addition, it is seen from Fig. 10 that $m_{s, 0}^{*}$ only has visible effects on the correlation between $J_{0}$ and $J_{0} / K_{0}$ for smaller $K_{0}$ values whereas it has almost no effect for larger $K_{0}$ values. Although there do not exist any empirical constraints on the $J_{0}$ parameter, we assume in the present study that the correlation between $J_{0}$ and $K_{0}$ from the MSL model is valid and then determine $J_{0} / K_{0}$ from the experimental constraint on $K_{0}$.

\section{Correlation between $L$ and $K_{\mathrm{sym}}$}

The parameters $L$ and $K_{\text {sym }}$ are determined by the density dependence of the symmetry energy around the normal nuclear density $\rho_{0}$. In recent years, significant progress has been made both experimentally and theoretically in extracting the information on the behaviors of nuclear symmetry energy at subsaturation density. By using the isospin- and momentum-dependent IBUU04 transport model with in-medium $N N$ cross sections, the isospin diffusion data were found to be consistent with the symmetry energy from the MDI interaction with $x$ between 0 and -1 , which can be parametrized by a density-dependent symmetry energy of $E_{\text {sym }}(\rho) \approx 31.6\left(\rho / \rho_{0}\right)^{\gamma}$ with $\gamma=0.69-$ 1.05 at subnormal density $\left(\rho \leqslant \rho_{0}\right)[50,55,56]$, leading to the extraction of $61 \leqslant L \leqslant 111 \mathrm{MeV}$ and $-82 \leqslant K_{\text {sym }} \leqslant$ $101 \mathrm{MeV}[50,55,56]$. By using the Skyrme interactions consistent with the EOS obtained from the MDI interaction with $x$ between 0 and -1 , the neutron-skin thickness of heavy nuclei calculated within the Hartree-Fock approach is consistent with available experimental data [50,126] and also that from a relativistic mean-field model based on an accurately calibrated parameter set that reproduces the GMR in ${ }^{90} \mathrm{Zr}$ and ${ }^{208} \mathrm{~Pb}$ as well as the isovector giant dipole resonance of ${ }^{208} \mathrm{~Pb}$ [127]. The extracted symmetry energy further agrees with the symmetry energy $E_{\text {sym }}(\rho)=31.6\left(\rho / \rho_{0}\right)^{0.69}$ recently obtained from the isoscaling analyses of isotope ratios in intermediate-energy heavy-ion collisions [57], which gives $L \approx 65 \mathrm{MeV}$ and $K_{\text {sym }} \approx-61 \mathrm{MeV}$. Furthermore, the limited range of $E_{\mathrm{sym}}(\rho)$ at subsaturation density is essentially consistent with the symmetry energy $E_{\text {sym }}(\rho)=$ $12.5\left(\rho / \rho_{0}\right)^{2 / 3}+17.6\left(\rho / \rho_{0}\right)^{\gamma}$ with $\gamma=0.4-1.05$, extracted very recently from the ImQMD (improved QMD) model analyses of both the isospin diffusion data and the double neutron/proton ratio [59]. The symmetry energy $E_{\text {sym }}(\rho)=$ $12.5\left(\rho / \rho_{0}\right)^{2 / 3}+17.6\left(\rho / \rho_{0}\right)^{\gamma}$ with $\gamma=0.4-1.05$ leads to the constraints of $46 \leqslant L \leqslant 80 \mathrm{MeV}$ and $-63 \leqslant K_{\text {sym }} \leqslant-17 \mathrm{MeV}$.

It should be noted that all these constraints on $L$ and $K_{\text {sym }}$ are based on some unique energy density functionals and thus special correlation between $L$ and $K_{\text {sym }}$ has been implicitly assumed. It is thus of interest to study whether there exists a universal correlation between $L$ and $K_{\text {sym }}$ in different models. For the MDI interaction, it is implied from Eq. (45) that $L$ and $K_{\text {sym }}$ both change linearly with the parameter $x$. Therefore they are linearly correlated by varying the parameter $x$ that changes the density dependence of the symmetry energy. For the MSL model, we can obtain from Eq. (66) the following expressions:

$$
\begin{aligned}
L= & 2 E_{\mathrm{sym}}^{\mathrm{kin}}\left(\rho_{0}\right)+5 D+3\left[E_{\mathrm{sym}}\left(\rho_{0}\right)-E_{\mathrm{sym}}^{\mathrm{kin}}\left(\rho_{0}\right)-D\right] \\
& +3 y\left(\gamma_{\mathrm{sym}}-1\right)\left[E_{\mathrm{sym}}\left(\rho_{0}\right)-E_{\mathrm{sym}}^{\mathrm{kin}}\left(\rho_{0}\right)-D\right], \\
K_{\mathrm{sym}}= & 9 y \gamma_{\mathrm{sym}}\left(\gamma_{\mathrm{sym}}-1\right)\left[E_{\mathrm{sym}}\left(\rho_{0}\right)-E_{\mathrm{sym}}^{\mathrm{kin}}\left(\rho_{0}\right)-D\right] \\
& +10 D-2 E_{\mathrm{sym}}^{\mathrm{kin}}\left(\rho_{0}\right) .
\end{aligned}
$$




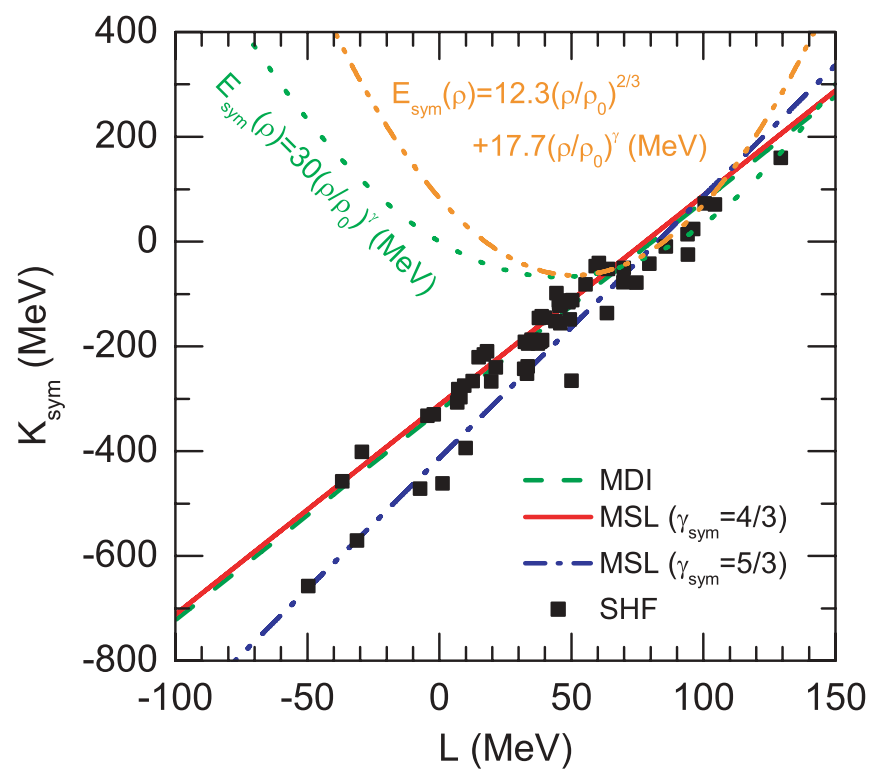

FIG. 11. (Color online) Correlation between $K_{\text {sym }}$ and $L$ from the MSL model with $\gamma_{\text {sym }}=4 / 3$ and 5/3, the MDI interaction, and the SHF prediction with the 63 Skyrme interactions shown in Table II. The results from two simple one-parameter symmetry energies in Eqs. (81) and (82) are also shown for comparison.

Therefore, for the MSL model, Eq. (78) and Eq. (79) show that $L$ and $K_{\text {sym }}$ both change linearly with the parameter $y$, and thus they are also linearly correlated by varying the parameter $y$ to change the density dependence of the symmetry energy. In particular, we have

$$
\begin{aligned}
K_{\mathrm{sym}}= & 3 \gamma_{\mathrm{sym}} L+E_{\mathrm{sym}}^{\mathrm{kin}}\left(\rho_{0}\right)\left(3 \gamma_{\mathrm{sym}}-2\right) \\
& +2 D\left(5-3 \gamma_{\mathrm{sym}}\right)-9 \gamma_{\mathrm{sym}} E_{\mathrm{sym}}\left(\rho_{0}\right) .
\end{aligned}
$$

Also, $L$ and $K_{\text {sym }}$ are expected to be correlated within the SHF energy density functional.

Shown in Fig. 11 are the correlation between $K_{\text {sym }}$ and $L$ from the MSL model with $\gamma_{\mathrm{sym}}=4 / 3$ and $5 / 3$ [in which we used the default values of $E_{\mathrm{sym}}^{\mathrm{kin}}\left(\rho_{0}\right)=12.3 \mathrm{MeV}, E_{\mathrm{sym}}\left(\rho_{0}\right)=$ $30 \mathrm{MeV}$, and $D=-3.51 \mathrm{MeV}$ from $m_{s, 0}^{*}=0.8 m$ and $m_{v, 0}^{*}=$ $0.7 \mathrm{~m}$ ], from the MDI interaction, and from the SHF prediction with the 63 Skyrme interactions shown in Table II. It is seen that the $K_{\text {sym }}$ parameter indeed displays approximately a linear correlation with the $L$ parameter for the SHF predictions with the 63 Skyrme interactions and this linear correlation is nicely reproduced by the MDI interaction and the MSL model with $\gamma_{\text {sym }}=4 / 3$. For the MSL model, one can see from Eq. (80) that the $\gamma_{\text {sym }}$ parameter controls the shape (slope) of the linear correlation between $L$ and $K_{\text {sym }}$. Furthermore, Fig. 11 shows that results from some Skyrme interactions deviate from the linear correlation obtained by the MDI interaction and the MSL model with $\gamma_{\mathrm{sym}}=4 / 3$. To consider the uncertainty in the shape (slope) for the correlation between $L$ and $K_{\text {sym }}$, we also include the result from the MSL model with $\gamma_{\text {sym }}=5 / 3$. The correlation between $K_{\text {sym }}$ and $L$ from the SHF predictions with the 63 Skyrme interactions is consistent with that from the MSL model with $\gamma_{\text {sym }}=4 / 3$ and 5/3. Furthermore, we find that the isospin-dependent nucleon effective mass has a very small effect on the correlation between $K_{\text {sym }}$ and $L$. This can be easily understood from Eq. (80) since the value of $2 D\left(5-3 \gamma_{\text {sym }}\right)$ is only about $7 \mathrm{MeV}$ for $\gamma_{\text {sym }}=4 / 3$ (and is zero for $\gamma_{\text {sym }}=5 / 3$ ). The linear correlation between $K_{\text {sym }}$ and $L$ implies that one can extract $K_{\text {sym }}$ from $L$.

As previously mentioned, a one-parameter parametrization for the symmetry energy is sometimes used for simplicity, that is,

$$
E_{\text {sym }}(\rho)=E_{\text {sym }}\left(\rho_{0}\right)\left(\frac{\rho}{\rho_{0}}\right)^{\gamma}
$$

or

$$
\begin{aligned}
E_{\mathrm{sym}}(\rho)= & E_{\mathrm{sym}}^{\mathrm{kin}}\left(\rho_{0}\right)\left(\frac{\rho}{\rho_{0}}\right)^{2 / 3} \\
& +\left[E_{\mathrm{sym}}\left(\rho_{0}\right)-E_{\mathrm{sym}}^{\mathrm{kin}}\left(\rho_{0}\right)\right]\left(\frac{\rho}{\rho_{0}}\right)^{\gamma} .
\end{aligned}
$$

For the parametrization of Eq. (81), $L$ and $K_{\text {sym }}$ can be obtained as

$$
\begin{aligned}
L & =3 \gamma E_{\mathrm{sym}}\left(\rho_{0}\right), \\
K_{\mathrm{sym}} & =9 \gamma(\gamma-1) E_{\mathrm{sym}}\left(\rho_{0}\right),
\end{aligned}
$$

and furthermore we have

$$
K_{\text {sym }}=\frac{L^{2}}{E_{\text {sym }}\left(\rho_{0}\right)}-3 L,
$$

which indicates that the $K_{\text {sym }}$ parameter is quadratically correlated with $L$. The result obtained by varying the parameter $\gamma$ is shown in Fig. 11 by the dotted line. Similarly, for the parametrization of Eq. (82), $L$ and $K_{\text {sym }}$ can be expressed as

$$
\begin{aligned}
L= & 2 E_{\mathrm{sym}}^{\mathrm{kin}}\left(\rho_{0}\right)+3 \gamma\left[E_{\mathrm{sym}}\left(\rho_{0}\right)-E_{\mathrm{sym}}^{\mathrm{kin}}\left(\rho_{0}\right)\right], \\
K_{\mathrm{sym}}= & 9 \gamma(\gamma-1)\left[E_{\mathrm{sym}}\left(\rho_{0}\right)-E_{\mathrm{sym}}^{\mathrm{kin}}\left(\rho_{0}\right)\right] \\
& -2 E_{\mathrm{sym}}^{\mathrm{kin}}\left(\rho_{0}\right),
\end{aligned}
$$

and the $K_{\text {sym }}$ parameter is again quadratically correlated with $L$, and this is illustrated by the dash-dot-dotted line in Fig. 11 obtained by varying the value of $\gamma$.

It is very interesting to see from Fig. 11 that for larger $L$ values ( $L \geqslant 45 \mathrm{MeV}$, which is consistent with the constraint from heavy-ion collision data shown later), all these symmetry energy functionals from different models and parametrizations give consistent predictions for the $K_{\mathrm{sym}}-L$ correlation. This nice feature implies that using these different models and parametrizations for the symmetry energy will not influence significantly the determination of the $K_{\text {sat, } 2}$ parameter. However, the $K_{\mathrm{sym}}-L$ correlation from the two one-parameter parametrizations on the symmetry energies in Eqs. (81) and (82) deviate significantly from the MDI interaction, MSL, and SHF predictions for small $L$ values. Actually, the two forms of one-parameter parametrization for the symmetry energy in Eqs. (81) and (82) may be too simple to describe a softer symmetry energy. As shown in Ref. [55], Eq. (81) cannot describe correctly the density dependence of the symmetry energy from the MDI interaction with $x=1$ (the Gogny interaction). However, as shown in Fig. 4, the MSL model can give a nice description of the density dependence of the symmetry energy from the very soft to the very stiff. Although 


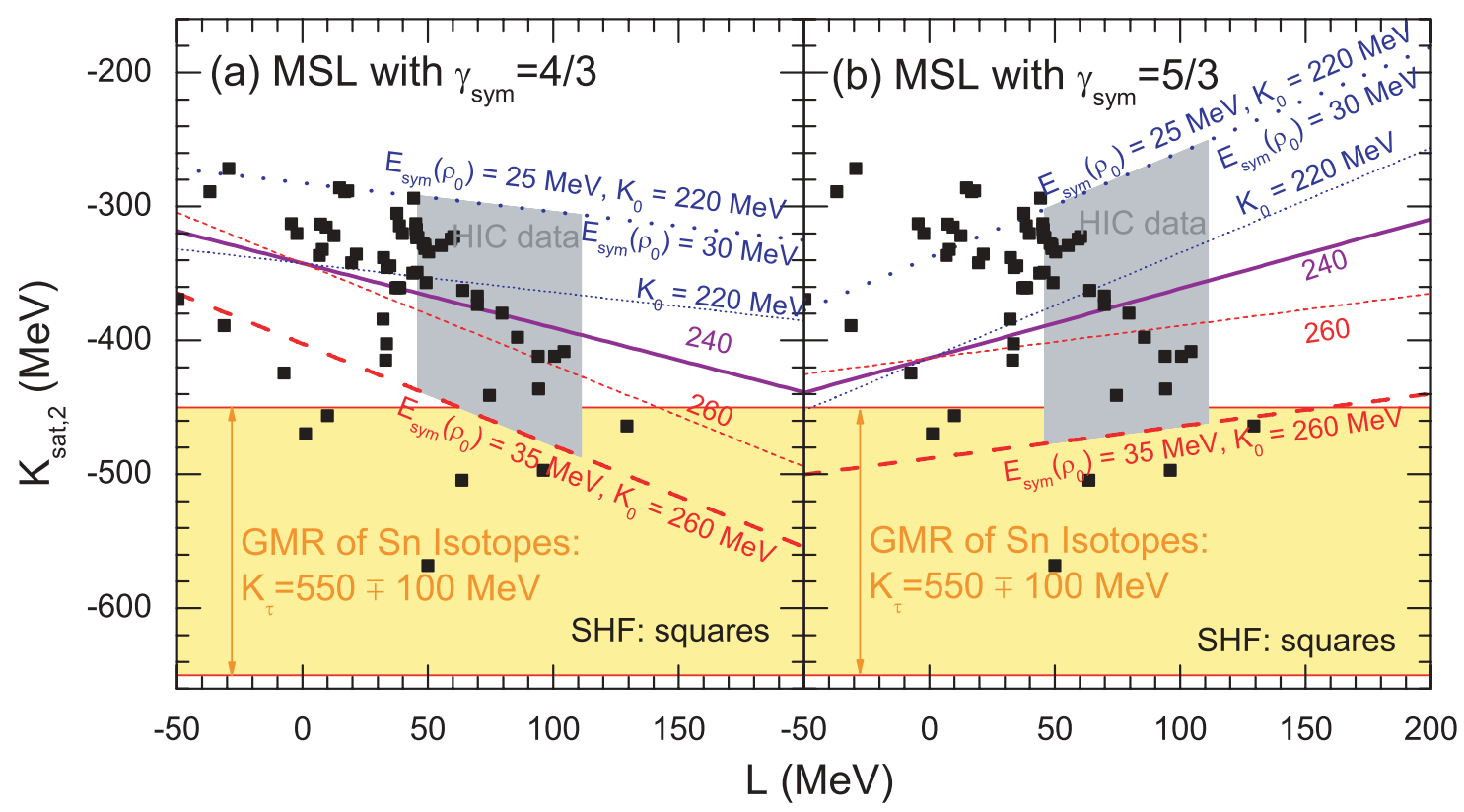

FIG. 12. (Color online) $K_{\mathrm{sat}, 2}$ as a function of $L$ from the MSL model with (a) $\gamma_{\mathrm{sym}}=4 / 3$ and (b) $5 / 3$ and $m_{s, 0}^{*}=0.8 m$ and $m_{v, 0}^{*}=0.7 m$ for different values of $K_{0}$ and $E_{\text {sym }}\left(\rho_{0}\right)$. The shaded region indicates constraints within the MSL model with $K_{0}=240 \pm 20 \mathrm{MeV}, E_{\mathrm{sym}}\left(\rho_{0}\right)=30 \pm$ $5 \mathrm{MeV}$, and $46 \leqslant L \leqslant 111 \mathrm{MeV}$ limited by the heavy-ion collision data. The results from the SHF approach with 63 Skyrme interactions are also included for comparison. In addition, the constraint of $K_{\tau}=-550 \pm 100 \mathrm{MeV}$ obtained in Refs. [22,23] from measurements of the isotopic dependence of the GMR in even-A Sn isotopes is also indicated.

there is no direct empirical information on the $K_{\text {sym }}$ parameter and some uncertainty on the $K_{\text {sym }}-L$ correlation still exists, we assume here that the correlation between $K_{\text {sym }}$ and $L$ from the MSL model with $\gamma_{\text {sym }}=4 / 3$ and 5/3 is valid and then use the experimental constraint on $L$ to extract the value of $K_{\text {sym }}$.

\section{Phenomenological MSL model constraint on the $K_{\text {sat,2 }}$ parameter}

We can now extract information on the $K_{\text {sat,2 }}$ parameter from the experimental constraints on the $K_{0}$ parameter and the $L$ parameter within the phenomenological MSL model. As pointed out previously, the value of $K_{0}$ has been relatively well determined to be $240 \pm 20 \mathrm{MeV}$ from the nuclear GMR [16,21-24]. The slope parameter $L$ has been found to correlate linearly with the neutron-skin thickness of heavy nuclei and thus can in principle be determined from measured thickness of the neutron skin of such nuclei [50,64,126,128-132]. Unfortunately, because of the large uncertainties in the experimental measurements, this has not yet been possible so far. The proposed experiment of parity-violating electron scattering from ${ }^{208} \mathrm{~Pb}$ [i.e., the Parity Radius Experiment (PREx) at the Jefferson Laboratory] is expected to give an independent and accurate measurement of its neutron skin thickness (within $0.05 \mathrm{fm})[133,134]$ and thus to impose a stringent constraint on the slope parameter $L$ in future. However, as mentioned previously, heavy-ion collisions, especially those induced by neutron-rich nuclei, provide a unique tool to explore the density dependence of the symmetry energy and thus the $L$ parameter. Actually, as discussed previously, the $L$ parameter has been already limited significantly by heavy-ion collision data.

From Eqs. (35) and (80), we have in the MSL model

$$
\begin{aligned}
K_{\mathrm{sat}, 2}= & K_{\mathrm{sym}}-6 L-\frac{J_{0}}{K_{0}} L=-\left(\frac{J_{0}}{K_{0}}+6-3 \gamma_{\mathrm{sym}}\right) L \\
& +\left(3 \gamma_{\mathrm{sym}}-2\right) E_{\mathrm{sym}}^{\mathrm{kin}}\left(\rho_{0}\right)+2 D\left(5-3 \gamma_{\mathrm{sym}}\right) \\
& -9 \gamma_{\mathrm{sym}} E_{\mathrm{sym}}\left(\rho_{0}\right) .
\end{aligned}
$$

Results on $K_{\text {sat,2 }}$ as a function of $L$ are shown in Fig. 12 for $\gamma_{\text {sym }}=4 / 3$ [Fig. 12(a)] and 5/3 [Fig. 12(b)] and with $K_{0}=220,240$, and $260 \mathrm{MeV}$. In Eq. (86), the value of $J_{0}$ is obtained from Eq. (74) by using the value of $K_{0}$. For other quantities in the MSL model, the default values $E_{\mathrm{sym}}^{\mathrm{kin}}\left(\rho_{0}\right)=12.3 \mathrm{MeV}, E_{\mathrm{sym}}\left(\rho_{0}\right)=30 \mathrm{MeV}$, and $D=$ $-3.51 \mathrm{MeV}$ from $m_{s, 0}^{*}=0.8 m$ and $m_{v, 0}^{*}=0.7 m$ have been used in the calculations. Since the value of $2 D\left(5-3 \gamma_{\text {sym }}\right)$ is only about $7 \mathrm{MeV}$ for $\gamma_{\mathrm{sym}}=4 / 3$ and is zero for $\gamma_{\mathrm{sym}}=5 / 3$, as mentioned before, the contribution of the $D$ parameter to $K_{\mathrm{sat}, 2}$ is thus small. Also, the correlation between $K_{\text {sym }}$ and $L$ depends on $E_{\text {sym }}\left(\rho_{0}\right)$ and $D$ as shown in Eq. (80). To take into consideration the uncertainty in the value of $E_{\text {sym }}\left(\rho_{0}\right)$, we also include in Fig. 12 the results with $K_{0}=$ $220 \mathrm{MeV}$ and $E_{\text {sym }}\left(\rho_{0}\right)=25 \mathrm{MeV}$ as well as $K_{0}=260 \mathrm{MeV}$ and $E_{\text {sym }}\left(\rho_{0}\right)=35 \mathrm{MeV}$, which represent, respectively, the upper and lower bounds for a fixed value of $L$. The shaded region in Fig. 12 further shows the constrained $L$ values from heavy-ion collision data, namely, $46 \leqslant L \leqslant 111 \mathrm{MeV}$. The lower limit of $L=46 \mathrm{MeV}$ is obtained from the lower bound in the ImQMD analyses of the isospin diffusion data and the double neutron/proton ratio [59] whereas the upper limit of 
$L=111 \mathrm{MeV}$ corresponds to the upper bound of $L$ from the IBUU04 transport model analysis of the isospin diffusion data [50,54-56]. The constraint $46 \leqslant L \leqslant 111 \mathrm{MeV}$ is consistent with the analyses of the pygmy dipole resonances [66], the giant dipole resonance (GDR) of ${ }^{208} \mathrm{~Pb}$ analyzed with Skyrme interactions [65], the very precise Thomas-Fermi model fit to the binding energies of 1654 nuclei [63], and the recent neutron-skin analysis [78]. These empirically extracted values of $L$ represent the best and most stringent phenomenological constraints available so far on the nuclear symmetry energy at subsaturation densities.

It is seen from Fig. 12 that the $K_{\text {sat,2 }}$ parameter decreases with increasing $L$ for $\gamma_{\text {sym }}=4 / 3$ whereas the opposite behavior is observed for $\gamma_{\mathrm{sym}}=5 / 3$. This feature can be easily understood from Eq. (86). For $\gamma_{\text {sym }}=4 / 3$, Eq. (86) is reduced to

$$
K_{\mathrm{sat}, 2}=-\left(\frac{J_{0}}{K_{0}}+2\right) L-12 E_{\mathrm{sym}}\left(\rho_{0}\right)+17.6(\mathrm{MeV}),
$$

and for $\gamma_{\text {sym }}=5 / 3$, it is reduced to

$$
K_{\mathrm{sat}, 2}=-\left(\frac{J_{0}}{K_{0}}+1\right) L-15 E_{\mathrm{sym}}\left(\rho_{0}\right)+36.9(\mathrm{MeV}) .
$$

For $K_{0}=240 \pm 20 \mathrm{MeV}$, Fig. 10 [or Eq. (74)] shows that the value of $J_{0} / K_{0}$ varies from about -1.9 to -1.2 when $m_{s, 0}^{*}$ changes from $m$ to $0.7 m$. Equation (87) [Eq. (88)] thus shows that $K_{\mathrm{sat}, 2}$ decreases (increases) with increasing $L$ for $\gamma_{\text {sym }}=4 / 3(5 / 3)$.

An interesting feature observed from Fig. 12 is that the $K_{\text {sat,2 }}$ parameter depends significantly on the symmetry energy at normal nuclear density, $E_{\mathrm{sym}}\left(\rho_{0}\right)$. This can be seen more clearly from Eqs. (87) and (88), which show that changing $E_{\text {sym }}\left(\rho_{0}\right)$ by $5 \mathrm{MeV}$ leads to a variation of 60 to $75 \mathrm{MeV}$ for $K_{\text {sat }, 2}$. This feature indicates that an accurate determination of $E_{\text {sym }}\left(\rho_{0}\right)$ is important for determining the value of $K_{\text {sat }, 2}$. In addition, we have used $E_{\text {sym }}\left(\rho_{0}\right)=30 \mathrm{MeV}$ in Fig. 11 and one can easily see that a variation of $5 \mathrm{MeV}$ for $E_{\text {sym }}\left(\rho_{0}\right)$ would lead to a shift of 60 to $75 \mathrm{MeV}$ for the $K_{\text {sym }}$ parameter for a fixed $L$. Therefore, the MSL model with $\gamma_{\text {sym }}=4 / 3$ and $5 / 3$ together with $E_{\text {sym }}\left(\rho_{0}\right)=30 \pm 5 \mathrm{MeV}$ may provide a nice estimate for both the $K_{\mathrm{sym}}-L$ correlation and its uncertainty.

From the shaded region indicated in Fig. 12, we find that for $\gamma_{\mathrm{sym}}=4 / 3$, we have $-437 \leqslant K_{\mathrm{sat}, 2} \leqslant-292 \mathrm{MeV}$ for $L=$ $46 \mathrm{MeV}$ and $-487 \leqslant K_{\text {sat }, 2} \leqslant-306 \mathrm{MeV}$ for $L=111 \mathrm{MeV}$. For $\gamma_{\text {sym }}=5 / 3$, we have $-477 \leqslant K_{\text {sat }, 2} \leqslant-302 \mathrm{MeV}$ for $L=$ $46 \mathrm{MeV}$ and $-461 \leqslant K_{\mathrm{sat}, 2} \leqslant-251 \mathrm{MeV}$ for $L=$ $111 \mathrm{MeV}$. These results indicate that based on the MSL model with $4 / 3 \leqslant \gamma_{\text {sym }} \leqslant 5 / 3, K_{0}=240 \pm 20 \mathrm{MeV}, 25 \leqslant E_{\text {sym }}\left(\rho_{0}\right) \leqslant$ $35 \mathrm{MeV}$, and $46 \leqslant L \leqslant 111 \mathrm{MeV}$, the $K_{\text {sat }, 2}$ parameter can vary from -251 to $-487 \mathrm{MeV}$. The results shown in Fig. 12 are obtained from a $J_{0} / K_{0}$ value that is evaluated with the default value $m_{s, 0}^{*}=0.8 m$. Similar analyses indicate that the $K_{\text {sat,2 }}$ parameter varies from -261 to $-489 \mathrm{MeV}$ if we use $m_{s, 0}^{*}=0.7 \mathrm{~m}$ whereas it varies from -245 to $-485 \mathrm{MeV}$ if $m_{s, 0}^{*}=0.9 m$ is used. These results indicate that the extracted value for $K_{\text {sat,2 }}$ is not sensitive to the variation of the nucleon effective mass. The MSL model analyses with $4 / 3 \leqslant \gamma_{\text {sym }} \leqslant 5 / 3, K_{0}=240 \pm$ $20 \mathrm{MeV}, E_{\text {sym }}\left(\rho_{0}\right)=30 \pm 5 \mathrm{MeV}, 46 \leqslant L \leqslant 111 \mathrm{MeV}$, and $m_{s, 0}^{*}=0.8 \pm 0.1 m$ thus lead to an estimate of $K_{\mathrm{sat}, 2}=$ $-370 \pm 120 \mathrm{MeV}$.

Also included in Fig. 12 are the results from the SHF approach with the 63 Skyrme interactions. It is seen that among the 63 Skyrme interactions shown in Table I, there are only 19 Skyrme interactions with predicted $K_{\text {sat,2 }}$ consistent with the constraints obtained here [the shaded regions in Fig. 12(a) or 12(b)]. The 19 Skyrme interactions are SKa, SKT4, $\mathrm{G}_{\sigma}, \mathrm{SkI} 3, \mathrm{SkI} 2, \mathrm{R}_{\sigma}, \mathrm{SKO}, \mathrm{SKO}^{*}, \mathrm{SkMP}, \mathrm{SGI}, \mathrm{SKM}, \mathrm{SLy} 5$, SLy1, SLy9, SLy2, SLy7, SkI6, SLy230b, and SkI4. From Tables I and II, one can see that all these 19 Skyrme interactions predict $0.151 \leqslant \rho_{0} \leqslant 0.161 \mathrm{fm}^{-3},-16.0 \leqslant E_{0}\left(\rho_{0}\right) \leqslant$ $-15.6 \mathrm{MeV}, 217 \leqslant K_{0} \leqslant 263 \mathrm{MeV}, 28 \leqslant E_{\text {sym }}\left(\rho_{0}\right) \leqslant 35 \mathrm{MeV}$, and $46 \leqslant L \leqslant 104 \mathrm{MeV}$, which are consistent with the empirical information. However, the value of the nucleon effective mass predicted by the 19 Skyrme interactions varies widely (i.e., from $0.58 m$ to $m$ for $m_{s, 0}^{*}$ and from $0.51 m$ to $m$ for $m_{v, 0}^{*}$ ). If we further impose the constraints of $m_{s, 0}^{*}=0.8 \pm 0.1 \mathrm{~m}$ and $m_{s, 0}^{*}>m_{v, 0}^{*}$, then only five Skyrme interactions, namely, $G_{\sigma}, R_{\sigma}, \mathrm{SKM}, \mathrm{SKO}$, and $\mathrm{SKO}^{*}$, remain to be consistent with all known empirical constraints except that the SKM interaction gives a little smaller incompressibility $K_{0}=$ $216.6 \mathrm{MeV}$ than the empirical constrained values of $K_{0}=$ $240 \pm 20 \mathrm{MeV}$. These features imply that the constraint on the $K_{\mathrm{sat}, 2}$ parameter does not significantly limit the nucleon effective mass as the former has previously been shown to be insensitive to the latter.

\section{Parameters $K_{\mathrm{sat}, 2}, K_{\mathrm{asy}}$, and $K_{\tau}$}

As shown in Eq. (38), the $K_{\text {asy }}$ parameter corresponds to the $K_{\text {sat,2 }}$ parameter when $J_{0}$ is zero; that is, the parabolic approximation to the EOS of symmetric nuclear matter [Eq. (10)] is valid. In the MSL model, a vanishing $J_{0}$ requires a $K_{0}$ value of about $340 \mathrm{MeV}$, which is significantly larger than the empirical value of $240 \pm 20 \mathrm{MeV}$. Since $J_{0} / K_{0} \approx-1.5$ for $K_{0}=240 \mathrm{MeV}$ in the MSL model, we have $K_{\text {sat }, 2} \approx K_{\text {asy }}+1.5 L$ or $K_{\text {asy }} \approx K_{\text {sat }, 2}-1.5 L$. Therefore, the difference between the $K_{\text {asy }}$ parameter and the $K_{\text {sat,2 }}$ parameter depends on the slope parameter $L$ of the symmetry energy and a larger $L$ value (stiffer symmetry energy) would lead to a larger difference. It should be stressed, however, that the $K_{\text {asy }}$ parameter is completely determined by the density dependence of symmetry energy regardless of the EOS of symmetric nuclear matter.

Based on the IBUU04 transport model analysis of the isospin diffusion data, a value of $K_{\text {asy }}=-500 \pm 50$ $\mathrm{MeV}$ has been extracted from the symmetry energy obtained by the MDI interaction with the $x$ parameter between 0 and -1 . The extracted $K_{\text {asy }}=-500 \pm 50 \mathrm{MeV}$ is essentially consistent with the symmetry energy extracted from the ImQMD model analyses of both the isospin diffusion data and the double neutron/proton ratio [59], which predict a $K_{\text {asy }}$ value from about -500 to $-340 \mathrm{MeV}$, and the symmetry energy $E_{\text {sym }}(\rho)=$ $31.6\left(\rho / \rho_{0}\right)^{0.69}$ obtained from the isoscaling analyses [57], which gives $K_{\text {asy }} \approx-453 \mathrm{MeV}$. Furthermore, the constraint $K_{\text {asy }}=-500 \pm 50 \mathrm{MeV}$ is consistent with the very recent 
constraint of $K_{\text {asy }} \approx-500_{-100}^{+125} \mathrm{MeV}$ obtained from the study of neutron skin of finite nuclei [78]. In the MDI interaction, one finds $J_{0} / K_{0}=-2.1$ from Table I and we thus have $-311 \leqslant K_{\mathrm{sat}, 2} \leqslant-316 \mathrm{MeV}$, corresponding to the prediction from the MDI interaction with the $x$ parameter between 0 and -1 . Therefore, the $K_{\text {sat,2 }}$ value for the MDI interaction is significantly larger than the $K_{\text {asy }}$ value and is insensitive to the density dependence of symmetry energy. However, the $J_{0}$ parameter in the MDI interaction is important for the determination of the $K_{\mathrm{sat}, 2}$ parameter. The importance of the higher order $J_{0}$ parameter to $K_{\mathrm{sat}, 2}$ can be further seen from Fig. 9, which shows that for the 63 Skyrme interactions considered here, the magnitude of the $K_{\text {asy }}$ parameter is generally larger than that of the $K_{\text {sat }, 2}$ parameter, especially, for the stiffer symmetry energies (larger $L$ values). These results indicate that the higher order $J_{0}$ contribution to $K_{\mathrm{sat}, 2}$ generally cannot be neglected.

In Fig. 12, the constraint $K_{\tau}=-550 \pm 100 \mathrm{MeV}$ obtained in Refs. [22,23] from recent measurements of the isotopic dependence of the GMR in even-A Sn isotopes is also shown. Although the estimate of $K_{\text {sat }, 2}=-370 \pm 120 \mathrm{MeV}$ obtained in the present work has small overlap with the constraint of $K_{\tau}=-550 \pm 100 \mathrm{MeV}$, the latter still has a significantly larger magnitude than the former. According to Fig. 12, there are only six Skyrme interactions, namely, SII, SIV, SV, SI, SkI5, and SIII, that predict $K_{\text {sat }, 2}=-550 \pm 100 \mathrm{MeV}$. As shown in Table I, the values for $K_{0}$ from SI, SII, SIII SIV, and SV are all larger than $305 \mathrm{MeV}$, which is obviously inconsistent with the empirical constraint of $K_{0}=240 \pm 20 \mathrm{MeV}$. For SkI5, we have $L=129.3 \mathrm{MeV}$, which is significantly larger than the empirical constraint of $46 \leqslant L \leqslant 111 \mathrm{MeV}$. Therefore, none of the 63 Skyrme interactions considered in the present work is consistent simultaneously with $K_{\text {sat }, 2}=-550 \pm$ $100 \mathrm{MeV}$ and the empirical constraints of $K_{0}=240 \pm$ $20 \mathrm{MeV}$ and $46 \leqslant L \leqslant 111 \mathrm{MeV}$. These features imply that the $K_{\tau}$ parameter extracted from Eq. (73) based on the GMR may

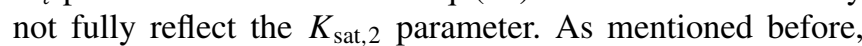
the $K_{\tau}$ parameter may depend on the detailed truncation scheme in Eq. (72). The constraint $K_{\tau}=-550 \pm 100 \mathrm{MeV}$ obtained in Refs. [22,23] is based on Eq. (73) and thus neglects contributions from the $K_{\text {curv }}$ term, $K_{\text {ss }}$, and other higher order terms in Eq. (72). It is expected that including contributions from higher order terms in Eq. (73) may change the extracted value for the $K_{\tau}$ parameter as found in Ref. [122].

\section{SUMMARY AND CONCLUSIONS}

We have studied in the present paper higher order effects on the properties of isospin asymmetric nuclear matter when the EOS and saturation properties of asymmetric nuclear matter are expanded in powers of the isospin asymmetry parameter $\delta=\left(\rho_{n}-\rho_{p}\right) / \rho$ and the dimensionless variable $\chi=\left(\rho-\rho_{0}\right) / 3 \rho_{0}$ that characterizes the deviations of the density from the normal nuclear density $\rho_{0}$. Analytical expressions for the saturation density of asymmetric nuclear matter as well as its binding energy and incompressibility at saturation density have been derived and given exactly up to fourth order in the isospin asymmetry $\delta=\left(\rho_{n}-\rho_{p}\right) / \rho$ using
11 characteristic parameters defined at normal nuclear density $\rho_{0}$ by the density derivatives of the binding energy per nucleon of symmetric nuclear matter, the symmetry energy $E_{\text {sym }}(\rho)$, and the fourth-order symmetry energy $E_{\mathrm{sym}, 4}(\rho)$, namely, $E_{0}\left(\rho_{0}\right), K_{0}, J_{0}, I_{0}, E_{\text {sym }}\left(\rho_{0}\right), L, K_{\text {sym }}, J_{\text {sym }}, E_{\text {sym }, 4}\left(\rho_{0}\right), L_{\text {sym }, 4}$, and $K_{\text {sym, } 4}$. Our method to derive the analytical expressions for the saturation properties of asymmetric nuclear matter is quite general, and in principle, the higher order coefficients (higher than $\delta^{4}$ ) in the isospin asymmetry $\delta$ can be easily obtained. Using the isospin- and momentum-dependent modified Gogy interaction, the SHF approach with 63 popular Skyrme interactions, and the phenomenological MSL model, we have systematically studied the higher order effects on the properties of asymmetric nuclear matter.

First, our results indicate that including terms up to $\chi^{4}$ can give a good description of the EOS of symmetric nuclear matter, $E_{0}(\rho)$, the density-dependent symmetry energy $E_{\text {sym }}(\rho)$, and the density-dependent fourth-order symmetry energy $E_{\text {sym }, 4}(\rho)$ for densities less than about $2 \rho_{0}$ whereas higher order terms in $\chi$ (higher than fourth order and thus requiring more characteristic parameters to be defined at $\rho_{0}$ ) are generally needed to describe reasonably $E_{0}(\rho), E_{\text {sym }}(\rho)$, and $E_{\text {sym }, 4}(\rho)$ in the higher density region (above $2 \rho_{0}$ ). These features imply that it is very difficult to predict the high-density behaviors of the EOS of asymmetric nuclear matter based on the characteristic parameters obtained at normal nuclear density $\rho_{0}$. Therefore, it is of great interest to use the transport model analysis of heavy-ion collisions at intermediate and high energies as well as the astrophysical observations of compact stars as tools to extract information on the EOS of asymmetric nuclear matter at high densities.

Second, we have systematically studied the isospin dependence of the saturation properties of asymmetric nuclear matter [i.e., the saturation density $\rho_{\text {sat }}(\delta)$ as well as the binding energy $E_{\text {sat }}(\delta)$ and incompressibility $K_{\text {sat }}(\delta)$ at saturation density]. In particular, we have compared their exact results with the analytical expressions expanded up to the $\delta^{2}$ term and the $\delta^{4}$ term, respectively. Our results indicate that the saturation properties of asymmetric nuclear matter [i.e., $\rho_{\text {sat }}(\delta), E_{\text {sat }}(\delta)$, and $\left.K_{\text {sat }}(\delta)\right]$ exhibit a good linear dependence on $\delta^{2}$ at smaller isospin asymmetries with $\delta^{2} \leqslant 0.1$, which is relevant to the structure of finite heavy nuclei. This feature implies that the higher order terms (the $\delta^{4}$ term and higher order terms in $\delta$ ) are not important for the saturation properties of asymmetric nuclear matter, at least for asymmetric nuclear matter that is not extremely neutron rich (proton rich). However, for asymmetric nuclear matter with extremely large isospin asymmetries with $\delta^{2} \geqslant 0.3$ and depending on the stiffness of the nuclear symmetry energy, the higher order terms in $\delta\left(\delta^{4}\right.$ and higher order terms) may become important for describing reasonably the saturation properties. It is further found that the saturation density and the incompressibility at saturation density generally decrease with the magnitude of the isospin asymmetry while the binding energy at saturation density shows the opposite behavior.

Finally, we have studied in detail the second-order isospin coefficient $K_{\text {sat,2 }}$ of the incompressibility of an asymmetric nuclear matter at its saturation density. It is found that the magnitude of the higher order $K_{\text {sat, } 4}$ parameter is generally 
small compared to that of the $K_{\text {sat,2 }}$ parameter, so the latter essentially characterizes the isospin dependence of the incompressibility at saturation density. Furthermore, we have found that the $K_{\text {sat,2 }}$ parameter is uniquely determined by $L, K_{\text {sym }}$, and $J_{0} / K_{0}$. Since there is no experimental information on the $J_{0}$ parameter and the $K_{\text {sym }}$ parameter, we have thus used the MSL model, which can reasonably describe the general properties of symmetric nuclear matter and the symmetry energy predicted by both the MDI interaction model and the SHF approach, to estimate the value of $K_{\text {sat,2. Our results }}$ indicate that generally the higher order $J_{0}$ contribution to $K_{\mathrm{sat}, 2}$ cannot be neglected, especially for larger $L$ values. Interestingly, it is found that there exists a nicely linear correlation between $K_{\mathrm{sym}}$ and $L$ as well as between $J_{0} / K_{0}$ and $K_{0}$ for the three different models used here (i.e., the MDI interaction, the MSL interaction, and the SHF approach with 63 Skyrme interactions). For the MSL model, the correlation between $K_{\text {sym }}$ and $L$ is further found to depend significantly on the value of $E_{\text {sym }}\left(\rho_{0}\right)$ but not on variations of the nucleon effective mass. These correlations and features have allowed us to extract the values of the $J_{0}$ parameter and the $K_{\text {sym }}$ parameter from the empirical information on $K_{0}, L$, and $E_{\text {sym }}\left(\rho_{0}\right)$. In particular, using the empirical constraints of $K_{0}=240 \pm$ $20 \mathrm{MeV}, E_{\text {sym }}\left(\rho_{0}\right)=30 \pm 5 \mathrm{MeV}, 46 \mathrm{M} \leqslant L \leqslant 111 \mathrm{MeV}$, and $m_{s, 0}^{*}=0.8 \pm 0.1 m$ in the MSL model leads to an estimate of $K_{\text {sat }, 2}=-370 \pm 120 \mathrm{MeV}$.

Although the estimated value of $K_{\text {sat, } 2}=-370 \pm 120 \mathrm{MeV}$ in the present work has small overlap with the constraint of $K_{\tau}=-550 \pm 100 \mathrm{MeV}$ obtained in Refs. [22,23] from recent measurements of the isotopic dependence of the GMR in even-A Sn isotopes, its magnitude is significantly smaller than that of the constrained $K_{\tau}$. Recently, there have been several studies $[118,135,136]$ on extracting the value of the $K_{\text {sat,2 }}$ parameter based on the idea initiated by Blaizot and collaborators that the values of both $K_{0}$ and $K_{\text {sat,2 }}$ should be extracted from the same consistent theoretical model that successfully reproduces the experimental GMR energies of a variety of nuclei. These studies show that no single model (interaction) can simultaneously describe correctly the recent measurements of the isotopic dependence of the GMR in even-A Sn isotopes and the GMR data of ${ }^{90} \mathrm{Zr}$ and ${ }^{208} \mathrm{~Pb}$ nuclei, and this makes it difficult to accurately determine the value of $K_{\mathrm{sat}, 2}$ from these experimental data. Also, a very recent study [137] indicates that nuclear superfluidity may also affect the extraction of the $K_{\text {sat,2 }}$ parameter from the nuclear GMR. As pointed out in Ref. [118], these features suggest that the $K_{\tau}=-550 \pm 100 \mathrm{MeV}$ value obtained in Refs. [22,23] may suffer from the same ambiguities already encountered in earlier attempts to extract the $K_{0}$ and $K_{\text {sat,2 }}$ values of infinite nuclear matter from finite-nuclei extrapolations. This problem remains an open challenge, and both experimental and theoretical insights are needed in future studies.

\section{ACKNOWLEDGMENTS}

We thank A. Rios for useful communications. This work was supported in part by the National Natural Science Foundation of China under Grant Nos. 10575071 and 10675082 ,
MOE of China under Project No. NCET-05-0392, Shanghai Rising-Star Program under Grant No. 06QA14024, the SRF for ROCS, SEM of China, the National Basic Research Program of China (973 Program) under Contract No. 2007CB815004, the US National Science Foundation under Grant Nos. PHY0652548, PHY-0757839, and PHY-0758115, the Welch Foundation under Grant No. A-1358, the Research Corporation under Grant No. 7123, and the Texas Coordinating Board of Higher Education Grant No. 003565-0004-2007.

\section{APPENDIX: DERIVATION OF ANALYTICAL EXPRESSIONS FOR THE SATURATION DENSITY AND INCOMPRESSIBILITY TO HIGHER ORDER IN ISOSPIN ASYMMETRY}

For completeness, we present in this Appendix a simple derivation of the analytical expressions for the saturation density of asymmetric nuclear matter and its incompressibility at saturation density up to higher order terms in the isospin asymmetry $\delta=\left(\rho_{n}-\rho_{p}\right) / \rho$. In particular, analytical expressions for the saturation density and incompressibility at saturation density are given exactly up to fourth order in $\delta$.

The binding energy per nucleon, $e(\rho, \delta)$, of asymmetric nuclear matter can be expanded up to $2 n$th order in $\delta$ as

$e(\rho, \delta)=e_{0}(\rho)+e_{2}(\rho) \delta^{2}+e_{4}(\rho) \delta^{4}+\cdots+e_{2 n}(\rho) \delta^{2 n}$.

The first term $e_{0}(\rho)$ represents the EOS of symmetric nuclear matter with $\delta=0$. We further expand each $e_{i}(\rho)$ in Eq. (A1) up to $n$th order in a dimensionless variable $z=\left(\rho-\rho_{0}\right) / \rho_{0}$, that is,

$$
\begin{aligned}
& e_{0}(\rho)=e_{0}\left(\rho_{0}\right)+a_{01} z+a_{02} z^{2}+\cdots+a_{0 n} z^{n}, \\
& e_{2}(\rho)=e_{2}\left(\rho_{0}\right)+a_{11} z+a_{12} z^{2}+\cdots+a_{1 n} z^{n}, \\
& e_{4}(\rho)=e_{4}\left(\rho_{0}\right)+a_{21} z+a_{22} z^{2}+\cdots+a_{2 n} z^{n}, \\
& \vdots \\
& e_{2 n}(\rho)=e_{2 n}\left(\rho_{0}\right)+a_{n 1} z+a_{n 2} z^{2}+\cdots+a_{n n} z^{n} .
\end{aligned}
$$

The coefficients $a_{i j}$ represent the characteristic parameters of asymmetric nuclear matter defined at normal nuclear density $\rho_{0}$. The saturation density $\rho_{\text {sat }}$ is then determined by the equation

$$
\left.\frac{\partial e}{\partial \rho}\right|_{\rho=\rho_{\text {sat }}}=0,
$$

or equivalently,

$$
\left.\frac{\partial e}{\partial z}\right|_{z=z_{\mathrm{sat}}}=0,
$$

with $z_{\text {sat }}=\left(\rho_{\text {sat }}-\rho_{0}\right) / \rho_{0}$

Substituting Eq. (A1) and Eq. (A2) into Eq. (A4) leads to the following equation:

$$
\begin{aligned}
a_{01}+ & 2 a_{02} z+3 a_{03} z^{2}+\cdots+n a_{0 n} z^{n-1} \\
& +\left(a_{11}+2 a_{12} z+3 a_{13} z^{2}+\cdots+n a_{1 n} z^{n-1}\right) \delta^{2} \\
& +\left(a_{21}+2 a_{22} z+3 a_{23} z^{2}+\cdots+n a_{2 n} z^{n-1}\right) \delta^{4}+\cdots \\
& +\left(a_{n 1}+2 a_{n 2} z+3 a_{n 3} z^{2}+\cdots+n a_{n n} z^{n-1}\right) \delta^{2 n}=0 .
\end{aligned}
$$


Obviously, we have $a_{01}=0$ as the saturation density of symmetric nuclear matter is determined by $\left.\frac{\partial e}{\partial \rho}\right|_{\delta=0, \rho=\rho_{0}}=0$. To obtain the solution to Eq. (A5) [i.e., $z_{\text {sat }}$ with a precision of $\left.O\left(\delta^{2 k+2}\right)\right]$, we assume

$$
z_{\text {sat }}=A_{2} \delta^{2}+A_{4} \delta^{4}+\cdots+A_{2 k} \delta^{2 k},
$$

where $A_{2 m}(m=1,2, \ldots, k)$ are coefficients to be determined from comparing the coefficients of $\delta^{2 j}$ terms $(j=1,2, \ldots, m)$ on the two sides of Eq. (A5) after substituting Eq. (A6) into Eq. (A5). For example, for $k=1$ (i.e., $z_{\text {sat }}=A_{2} \delta^{2}$ ), the coefficient $A_{2}$ can be determined by the following equality:

$$
2 a_{02} A_{2}+a_{11}=0 .
$$

Similarly, for $k=2$ (i.e., $z_{\text {sat }}=A_{2} \delta^{2}+A_{4} \delta^{4}$ ), the coefficient $A_{4}$ can be determined by

$$
2 a_{02} A_{4}+3 a_{03} A_{2}^{2}+2 a_{12} A_{2}+a_{21}=0 .
$$

Furthermore, for $k=3$ and 4 , the coefficients $A_{6}$ and $A_{8}$ can be determined, respectively, by

$$
\begin{aligned}
& 2 a_{02} A_{6}+6 a_{03} A_{2} A_{4}+4 a_{04} A_{2}^{3}+2 a_{12} A_{4}+3 a_{13} A_{2}^{2} \\
& \quad+2 a_{22} A_{2}+a_{31}=0
\end{aligned}
$$

and

$$
\begin{aligned}
& 2 a_{02} A_{8}+3 a_{03}\left(2 A_{2} A_{6}+A_{4}^{2}\right)+12 a_{04} A_{2}^{2} A_{4}+5 a_{05} A_{2}^{4} \\
& \quad+2 a_{12} A_{6}+6 a_{13} A_{2} A_{4}+4 a_{14} A_{2}^{3}+2 a_{22} A_{4} \\
& \quad+3 a_{23} A_{2}^{2}+2 a_{32} A_{2}+a_{41}=0 .
\end{aligned}
$$

From this analysis, the coefficient of the $\delta^{2 m}$ term, $A_{2 m}$, can be determined by all lower order coefficients $A_{2}, A_{4}, A_{2 m-2}$ $(m=1,2,3, \ldots, n)$. The $A_{2 m}$ obtained here are complete and precise up to the order of $\delta^{2 m}$, and the higher order characteristic parameters $a_{i j}$ do not contribute to $A_{2 m}$. To write down the general expressions for $A_{2 m}$, we define the following symbol:

$$
B_{n, m}=\sum A_{2}^{i_{1}} A_{4}^{i_{2}} \cdots A_{2 n}^{i_{n}},
$$

where we have $i_{1}, i_{2}, \ldots i_{n} \in\left\{0, N_{+}\right\}$and they satisfy the following conditions:

$$
\sum_{j=1}^{n} j i_{j}=m, \quad \sum_{j=1}^{n} i_{j}=n .
$$

Then the coefficient of the $\delta^{2 m}$ term satisfies the equation

$$
\sum_{j=0}^{m-1} \sum_{i=2}^{m-j+1} i a_{j i} B_{i-1, m-j}+a_{m 1}=0,
$$

from which we can obtain the coefficient $A_{2 m}$ as

$$
\begin{aligned}
A_{2 m}= & -\frac{1}{a_{02}}\left(\sum_{i=3}^{m+1} i a_{0 i} B_{i-1, m}\right. \\
& \left.+\sum_{j=1}^{m-1} \sum_{i=2}^{m-j+1} i a_{j i} B_{i-1, m-j}+a_{m 1}\right) .
\end{aligned}
$$

Therefore, one can obtain the saturation density of asymmetric nuclear matter to any order of $\delta$.
Taking the precision to the order of $\delta^{4}$, we then have

$$
A_{2}=-\frac{a_{11}}{2 a_{02}}, \quad A_{4}=\frac{a_{12} a_{11}}{2 a_{02}^{2}}-\frac{3 a_{03} a_{11}^{2}}{8 a_{02}^{2}}-\frac{a_{21}}{2 a_{02}} .
$$

Converting the coefficients $a_{i j}$ into the conventional forms, that is,

$$
\begin{aligned}
& a_{02}=\frac{K_{0}}{18}, \quad a_{03}=\frac{J_{0}}{162}, \quad a_{04}=\frac{I_{0}}{24 \times 81}, \\
& a_{11}=\frac{L}{3}, \quad a_{12}=\frac{K_{\mathrm{sym}}}{18}, \quad a_{13}=\frac{J_{\mathrm{sym}}}{162}, \\
& a_{21}=\frac{L_{\mathrm{sym}, 4}}{3}, \quad a_{22}=\frac{K_{\mathrm{sym}, 4}}{18} .
\end{aligned}
$$

we then obtain

$z_{\mathrm{sat}}=-\frac{3 L}{K_{0}} \delta^{2}+\left(\frac{3 K_{\mathrm{sym}} L}{K_{0}^{2}}-\frac{3 L_{\mathrm{sym}, 4}}{K_{0}}-\frac{3 J_{0} L^{2}}{2 K_{0}^{3}}\right) \delta^{4}$.

So the saturation density can be obtained as

$$
\begin{aligned}
\rho_{\mathrm{sat}}= & \rho_{0}\left[1-\frac{3 L}{K_{0}} \delta^{2}\right. \\
& \left.+\left(\frac{3 K_{\mathrm{sym}} L}{K_{0}^{2}}-\frac{3 L_{\mathrm{sym}, 4}}{K_{0}}-\frac{3 J_{0} L^{2}}{2 K_{0}^{3}}\right) \delta^{4}+O\left(\delta^{6}\right)\right],
\end{aligned}
$$

which is exactly Eq. (22).

The incompressibility coefficient $K_{\text {sat }}$ of asymmetric nuclear matter at the saturation density is defined as

$$
K_{\text {sat }}=\left.9 \rho_{\text {sat }}^{2} \frac{\partial^{2} e}{\partial \rho^{2}}\right|_{\rho=\rho_{\text {sat }}} .
$$

Substituting Eq. (A1) and Eq. (A2) into Eq. (A19) and using Eq. (A18), we can easily obtain

$$
\begin{aligned}
K_{\mathrm{sat}}= & 9 \rho_{0}^{2}\left[1-\frac{3 L}{K_{0}} \delta^{2}+\left(\frac{3 K_{\mathrm{sym}} L}{K_{0}^{2}}-\frac{3 L_{\mathrm{sym}, 4}}{K_{0}}-\frac{3 J_{0} L^{2}}{2 K_{0}^{3}}\right) \delta^{4}\right]^{2} \\
& \times\left[\frac{K_{0}}{9 \rho_{0}^{2}}+\frac{J_{0}}{27 \rho_{0}^{2}} \frac{\rho-\rho_{0}}{\rho_{0}}+\frac{I_{0}}{162}\left(\frac{\rho-\rho_{0}}{\rho_{0}}\right)^{2}\right. \\
& +\left(\frac{K_{\mathrm{sym}}}{9 \rho_{0}^{2}}+\frac{J_{\mathrm{sym}}}{27 \rho_{0}^{2}} \frac{\rho-\rho_{0}}{\rho_{0}}+\frac{I_{\mathrm{sym}}}{162}\left(\frac{\rho-\rho_{0}}{\rho_{0}}\right)^{2}\right) \delta^{2} \\
& \left.+\left(\frac{K_{\mathrm{sym}, 4}}{9 \rho_{0}^{2}}+\frac{J_{\mathrm{sym}, 4}}{27 \rho_{0}^{2}} \frac{\rho-\rho_{0}}{\rho_{0}}\right) \delta^{4}+O\left(\delta^{6}\right)\right]\left.\right|_{\rho=\rho_{\mathrm{sat}}} \\
= & K_{0}+\left(K_{\mathrm{sym}}-6 L-\frac{J_{0}}{K_{0}} L\right) \delta^{2} \\
& +\left(K_{\mathrm{sym}, 4}-6 L_{\mathrm{sym}, 4}-\frac{J_{0} L_{\mathrm{sym}, 4}}{K_{0}}+\frac{9 L^{2}}{K_{0}}-\frac{J_{\mathrm{sym}} L}{K_{0}}\right. \\
& \left.+\frac{I_{0} L^{2}}{2 K_{0}^{2}}+\frac{J_{0} K_{\mathrm{sym}} L}{K_{0}^{2}}+\frac{3 J_{0} L^{2}}{K_{0}^{2}}-\frac{J_{0}^{2} L^{2}}{2 K_{0}^{3}}\right) \delta^{4} \\
& +O\left(\delta^{6}\right),
\end{aligned}
$$


which is exactly Eq. (33). We note that the expression $K_{\text {sat }}=K_{0}+\left(K_{\text {sym }}-6 L-\frac{J_{0}}{K_{0}} L\right) \delta^{2}$ was originally given in Ref. [119]. For terms higher than $\delta^{4}$, they can be straight- forwardly obtained following this derivation but many more higher order characteristic parameters $a_{i j}$ would be needed.
[1] W. Zhan et al., Int. J. Mod. Phys. E 15, 1941 (2006); http://www.impcas.ac.cn/zhuye/en/htm/247.htm.

[2] Y. Yano, Nucl. Instrum. Methods B 261, 1009 (2007).

[3] http://www.gsi.de/fair/index_e.html.

[4] http://www.ganil-spiral2.eu/research/developments/spiral2/.

[5] Whitepapers of the 2007 NSAC Long Range Plan Town Meeting, January, 2007, Chicago, http://dnp.aps.org.

[6] B. A. Li, C. M. Ko, and W. Bauer, Int. J. Mod. Phys. E 7, 147 (1998).

[7] B.-A. Li and W. U. Schröder (eds.), Isospin Physics in Heavy-Ion Collisions at Intermediate Energies (Nova Science, New York, 2001).

[8] P. Danielewicz, R. Lacey, and W. G. Lynch, Science 298, 1592 (2002).

[9] J. M. Lattimer and M. Prakash, Phys. Rep. 333-334, 121 (2000).

[10] J. M. Lattimer and M. Prakash, Astrophys. J. 550, 426 (2001).

[11] J. M. Lattimer and M. Prakash, Science 304, 536 (2004).

[12] V. Baran, M. Colonna, V. Greco, and M. Di Toro, Phys. Rep. 410, 335 (2005).

[13] A. W. Steiner, M. Prakash, J. M. Lattimer, and P. J. Ellis, Phys. Rep. 411, 325 (2005).

[14] L. W. Chen, C. M. Ko, B. A. Li, and G. C. Yong, Front. Phys. China 2, 327 (2007).

[15] B. A. Li, L. W. Chen, and C. M. Ko, Phys. Rep. 464, 113 (2008).

[16] D. H. Youngblood, H. L. Clark, and Y.-W. Lui, Phys. Rev. Lett. 82, 691 (1999).

[17] Y.-W. Lui, D. H. Youngblood, Y. Tokimoto, H. L. Clark, and B. John, Phys. Rev. C 70, 014307 (2004).

[18] Z. Y. Ma et al., Nucl. Phys. A703, 222 (2002).

[19] D. Vretenar, T. Niksic, and P. Ring, Phys. Rev. C 68, 024310 (2003).

[20] G. Colo, N. Van Giai, J. Meyer, K. Bennaceur, and P. Bonche, Phys. Rev. C 70, 024307 (2004).

[21] S. Shlomo, V. M. Kolomietz, and G. Colò, Eur. Phys. J. A 30, 23 (2006).

[22] T. Li et al., Phys. Rev. Lett. 99, 162503 (2007).

[23] U. Garg et al., Nucl. Phys. A788, 36 (2007).

[24] G. Colo, AIP Conf. Proc. 1128, 59 (2009).

[25] J. Aichelin and C. M. Ko, Phys. Rev. Lett. 55, 2661 (1985).

[26] C. Fuchs, Prog. Part. Nucl. Phys. 56, 1 (2006).

[27] W. D. Myers and W. J. Swiatecki, Nucl. Phys. 81, 1 (1966).

[28] K. Pomorski and J. Dudek, Phys. Rev. C 67, 044316 (2003).

[29] S. Ulrych and H. Müther, Phys. Rev. C 56, 1788 (1997).

[30] E. N. E. van Dalen, C. Fuchs, and A. Faessler, Nucl. Phys. A744, 227 (2004).

[31] Z. Y. Ma, J. Rong, B. Q. Chen, Z. Y. Zhu, and H. Q. Song, Phys. Lett. B604, 170 (2004).

[32] F. Sammarruca, W. Barredo, and P. Krastev, Phys. Rev. C 71, 064306 (2005)

[33] E. N. E. van Dalen, C. Fuchs, and A. Faessler, Phys. Rev. Lett. 95, 022302 (2005).

[34] E. N. E. van Dalen, C. Fuchs, and A. Faessler, Phys. Rev. C 72, 065803 (2005).

[35] J. Rong, Z. Y. Ma, and N. Van Giai, Phys. Rev. C 73, 014614 (2006).
[36] I. Bombaci and U. Lombardo, Phys. Rev. C 44, 1892 (1991).

[37] W. Zuo, L. G. Cao, B. A. Li, U. Lombardo, and C. W. Shen, Phys. Rev. C 72, 014005 (2005).

[38] Z. H. Li et al., Phys. Rev. C 74, 047304 (2006).

[39] M. Baldo and C. Maieron, J. Phys. G 34, R243 (2007).

[40] Z. Z. Ren, F. Tai, and D. H. Chen, Phys. Rev. C 66, 064306 (2002).

[41] J. Meng, H. Toki, S. G. Zhoub, S. Q. Zhang, W. H. Long, and L. S. Geng, Prog. Part. Nucl. Phys. 57, 470 (2006).

[42] L. W. Chen, C. M. Ko, and B. A. Li, Phys. Rev. C 76, 054316 (2007).

[43] C. B. Das, S. Das Gupta, C. Gale, and B. A. Li, Phys. Rev. C 67, 034611 (2003).

[44] B. A. Li, C. B. Das, S. Das Gupta, and C. Gale, Phys. Rev. C 69, 011603(R) (2004); Nucl. Phys. A735, 563 (2004).

[45] B. A. Li, Phys. Rev. C 69, 064602 (2004).

[46] L. W. Chen, C. M. Ko, and B. A. Li, Phys. Rev. C 69, 054606 (2004).

[47] J. Rizzo, M. Colonna, M. Di Toro, and V. Greco, Nucl. Phys. A732, 202 (2004).

[48] B. Behera, T. R. Routray, A. Pradhan, S. K. Patra, and P. K. Sahu, Nucl. Phys. A753, 367 (2005).

[49] J. Rizzo, M. Colonna, and M. Di Toro, Phys. Rev. C 72, 064609 (2005).

[50] L. W. Chen, C. M. Ko, and B. A. Li, Phys. Rev. C 72, 064309 (2005).

[51] L. W. Chen, Sci. China Ser. G (2009, in press).

[52] I. Bombaci, in Ref. [7], p. 35.

[53] S. Kubis and M. Kutschera, Nucl. Phys. A720, 189 (2003).

[54] M. B. Tsang et al., Phys. Rev. Lett. 92, 062701 (2004).

[55] L. W. Chen, C. M. Ko, and B. A. Li, Phys. Rev. Lett. 94, 032701 (2005).

[56] B. A. Li and L. W. Chen, Phys. Rev. C 72, 064611 (2005).

[57] D. V. Shetty, S. J. Yennello, and G. A. Souliotis, Phys. Rev. C 75, 034602 (2007).

[58] Y. Zhang et al., Phys. Lett. B664, 145 (2008).

[59] M. B. Tsang et al., Phys. Rev. Lett. 102, 122701 (2009).

[60] W. Trautmann et al., Prog. Part. Nucl. Phys. 62, 425 (2009).

[61] W. Reisdorf et al., Nucl. Phys. A781, 459 (2007).

[62] Z. G. Xiao, B. A. Li, L. W. Chen, G. C. Yong, and M Zhang, Phys. Rev. Lett. 102, 062502 (2009).

[63] W. D. Myers and W. J. Swiatecki, Nucl. Phys. A601, 141 (1996); Phys. Rev. C 57, 3020 (1998).

[64] B. A. Brown, Phys. Rev. Lett. 85, 5296 (2000).

[65] L. Trippa, G. Colo, and E. Vigezzi, Phys. Rev. C 77, 061304(R) (2008).

[66] A. Klimkiewicz et al. (LAND Collaboration), Phys. Rev. C 76, 051603(R) (2007).

[67] P. J. Siemens, Nucl. Phys. A141, 225 (1970).

[68] O. Sjöberg, Nucl. Phys. A222, 161 (1974).

[69] I. E. Lagaris and V. R. Pandharipande, Nucl. Phys. A369, 470 (1981).

[70] F. S. Zhang and L. W. Chen, Chin. Phys. Lett. 18, 142 (2001).

[71] A. W. Steiner, Phys. Rev. C 74, 045808 (2006).

[72] J. Xu, L. W. Chen, B. A. Li, and H. R. Ma, Phys. Rev. C 79, 035802 (2009). 
[73] J. Xu, L. W. Chen, B. A. Li, and H. R. Ma, Astrophys. J. 697, 1549 (2009).

[74] M. Prakash and K. S. Bedell, Phys. Rev. C 32, 1118 (1985).

[75] M. Lopez-Quelle, S. Marcos, R. Niembro, A. Bouyssy, and N. Van Giai, Nucl. Phys. A483, 479 (1988).

[76] V. Baran, M. Colonna, M. Di Toro, V. Greco, and M. ZielinskaPfabé, and H. H. Wolter, Nucl. Phys. A703, 603 (2002).

[77] P. Danielewicz and J. Lee, Nucl. Phys. A818, 36 (2009).

[78] M. Centelles, X. Roca-Maza, X. Vinas, and M. Warda, Phys. Rev. Lett. 102, 122502 (2009).

[79] B. A. Li, G. C. Yong, and W. Zuo, Phys. Rev. C 71, 014608 (2005).

[80] B. A. Li, G. C. Yong, and W. Zuo, Phys. Rev. C 71, 044604 (2005).

[81] B. A. Li, L. W. Chen, G. C. Yong, and W. Zuo, Phys. Lett. B634, 378 (2006).

[82] G. C. Yong, B. A. Li, L. W. Chen, and W. Zuo, Phys. Rev. C 73, 034603 (2006).

[83] G. C. Yong, B. A. Li, and L. W. Chen, Phys. Rev. C 74, 064617 (2006).

[84] G. C. Yong, B. A. Li, and L. W. Chen, Phys. Lett. B650, 344 (2007).

[85] J. Xu, L. W. Chen, B. A. Li, and H. R. Ma, Phys. Rev. C 75, 014607 (2007).

[86] J. Xu, L. W. Chen, B. A. Li, and H. R. Ma, Phys. Lett. B650, 348 (2007).

[87] B. A. Li, L. W. Chen, H. R. Ma, J. Xu, and G. C. Yong, Phys. Rev. C 76, 051601(R) (2007).

[88] D. Vautherin and D. M. Brink, Phys. Rev. C 5, 626 (1972).

[89] M. Brack, C. Guet, and H.-B. Hakansson, Phys. Rep. 123, 275 (1985).

[90] J. Friedrich and P.-G. Reinhard, Phys. Rev. C 33, 335 (1986).

[91] E. Chabanat, P. Bonche, P. Haensel, J. Meyer, and R. Schaeffer, Nucl. Phys. A627, 710 (1997).

[92] E. Chabanat, P. Bonche, P. Haensel, J. Meyer, and R. Schaeffer, Nucl. Phys. A635, 231 (1998); A643, 441(E) (1998).

[93] B. A. Brown, Phys. Rev. C 58, 220 (1998).

[94] L. W. Chen and F. S. Zhang, High Energy Phys. Nucl. Phys. 23, 1197 (1999) (in Chinese).

[95] J. R. Stone, J. C. Miller, R. Koncewicz, P. D. Stevenson, and M. R. Strayer, Phys. Rev. C 68, 034324 (2003).

[96] J. R. Stone and P.-G. Reinhard, Prog. Part. Nucl. Phys. 58, 587 (2007); D. T. Khoa, H. S. Than, and D. C. Cuong, Phys. Rev. C 76, 014603 (2007).

[97] J. M. Pearson and S. Goriely, Phys. Rev. C 64, 027301 (2001).

[98] O. Bohigas, A. M. Lane, and J. Martorell, Phys. Rep. 51, 267 (1979).

[99] H. Krivine, J. Treiner, and O. Bohigas, Nucl. Phys. A336, 155 (1980).

[100] M. Farine, J. M. Pearson, and F. Tondeur, Nucl. Phys. A696, 396 (2001).

[101] P.-G. Reinhard, Nucl. Phys. A649, 305c (1999).

[102] D. Lunney, J. M. Pearson, and C. Thibault, Rev. Mod. Phys. 75, 1021 (2003).

[103] T. Marketin, D. Vretenar, and P. Ring, Phys. Rev. C 75, 024304 (2007).
[104] M. Samyn et al., Nucl. Phys. A700, 142 (2002).

[105] S. Goriely, M. Samyn, M. Bender, and J. M. Pearson, Phys. Rev. C 68, 054325 (2003).

[106] M. Samyn, S. Goriely, and J. M. Pearson, Nucl. Phys. A725, 69 (2003).

[107] M. Samyn, S. Goriely, M. Bender, and J. M. Pearson, Phys. Rev. C 70, 044309 (2004).

[108] S. Goriely et al., Nucl. Phys. A750, 425 (2005).

[109] M. Samyn, S. Goriely, and J. M. Pearson, Phys. Rev. C 72, 044316 (2005).

[110] S. Goriely, M. Samyn, and J. M. Pearson, Nucl. Phys. A773, 279 (2006).

[111] S. Goriely, M. Samyn, and J. M. Pearson, Phys. Rev. C 75, 064312 (2007).

[112] S. Goriely and J. M. Pearson, Phys. Rev. C 77, 031301(R) (2008).

[113] N. Chamel, S. Goriely, and J. M. Pearson, Nucl. Phys. A812, 72 (2008).

[114] T. Klahn et al., Phys. Rev. C 74, 035802 (2006).

[115] A. Akmal, V. R. Pandharipande, and D. G. Ravenhall, Phys. Rev. C 58, 1804 (1998).

[116] I. Bombaci, T. T. S. Kuo, and U. Lombardo, Phys. Rep. 242, 165 (1994).

[117] E. Baron, J. Cooperstein, and S. Kahana, Phys. Rev. Lett. 55, 126 (1985); Nucl. Phys. A440, 744 (1985).

[118] J. Piekarewicz and M. Centelles, Phys. Rev. C 79, 054311 (2009).

[119] J. P. Blaizot, Phys. Rep. 64, 171 (1980).

[120] J. P. Blaizot and B. Grammaticos, Nucl. Phys. A355, 115 (1981).

[121] M. M. Sharma et al., Phys. Rev. C 38, 2562 (1988).

[122] S. Shlomo and D. H. Youngblood, Phys. Rev. C 47, 529 (1993).

[123] C. Fuchs, A. Faessler, E. Zabrodin, and Y.-M. Zheng, Phys. Rev. Lett. 86, 1974 (2001).

[124] C. Hartnack, H. Oeschler, and J. Aichelin, Phys. Rev. Lett. 96, 012302 (2006).

[125] J. M. Pearson, Phys. Lett. B271, 12 (1991).

[126] A. W. Steiner and B. A. Li, Phys. Rev. C 72, 041601(R) (2005).

[127] B. G. Todd-Rutel and J. Piekarewicz, Phys. Rev. Lett. 95, 122501 (2005).

[128] S. Typel and B. A. Brown, Phys. Rev. C 64, 027302 (2001).

[129] C. J. Horowitz and J. Piekarewicz, Phys. Rev. Lett. 86, 5647 (2001); Phys. Rev. C 64, 062802(R) (2001); Phys. Rev. C 66, 055803 (2002).

[130] R. J. Furnstahl, Nucl. Phys. A706, 85 (2002).

[131] S. Karataglidis, K. Amos, B. A. Brown, and P. K. Deb, Phys. Rev. C 65, 044306 (2002).

[132] A. E. L. Dieperink, Y. Dewulf, D. Van Neck, M. Waroquier, and V. Rodin, Phys. Rev. C 68, 064307 (2003).

[133] C. J. Horowitz, S. J. Pollock, P. A. Souder, and R. Michaels, Phys. Rev. C 63, 025501 (2001).

[134] R. Michaels, P. A. Souder, and G. M. Urciuoli, http://hallaweb. jlab.org/parity/prex.

[135] H. Sagawa, S. Yoshida, G. M. Zeng, J. Z. Gu, and X. Z. Zhang, Phys. Rev. C 76, 034327 (2007).

[136] J. Piekraewicz, Phys. Rev. C 76, 031301(R) (2007).

[137] E. Khan, arXiv:0905.3335. 